\title{
Targeting Galectin 3 to Counteract Spike-Phase Uncoupling of Fast-Spiking Interneurons to Gamma Oscillations in Alzheimer's Disease
}

\section{Luis Enrique Arroyo-García}

Karolinska Institutet

\section{Sara Bachiller}

Lund University: Lunds Universitet

\section{Antonio Boza-Serrano}

Lunds Universitet

\section{Antonio Rodríguez-Moreno}

Universidad Pablo de Olavide

\section{Tomas Deierborg}

Lunds Universitet

Yuniesky Andrade-Talavera ( $\nabla$ yandtal@upo.es )

Universidad Pablo de Olavide https://orcid.org/0000-0002-5295-0169

\section{André Fisahn}

Karolinska Institutet

\section{Research article}

Keywords: Galectin-3, gamma oscillations, neuronal network dynamics, fast-spiking interneurons, Alzheimer's disease models, TD139, hippocampus, neuroinflammation

Posted Date: December 29th, 2021

DOI: https://doi.org/10.21203/rs.3.rs-1193198/v1

License: (a) (7) This work is licensed under a Creative Commons Attribution 4.0 International License. Read Full License 


\section{Abstract}

Background: Alzheimer's disease (AD) is a progressive multifaceted neurodegenerative disorder for which no disease-modifying treatment exists. Neuroinflammation is central to the pathology progression, with evidence suggesting that microglia-released galectin 3 (gal3) plays a pivotal role by amplifying neuroinflammation in AD. However, possible involvement of gal3 in the disruption of cognition-relevant neuronal network oscillations typical of $A D$ remains unknown.

Methods: Here, we investigate the functional implications of gal3 signaling on cognition-relevant gamma oscillations $(30-80 \mathrm{~Hz})$ by performing electrophysiological recordings in hippocampal area CA3 of wild-type (WT) and 5xFAD mice in vitro.

Results: Gal3 application decreases gamma oscillation power and rhythmicity in an activity-dependent manner and is accompanied by impairment of cellular dynamics in fast-spiking interneurons (FSN) and pyramidal cells (PCs). We found that gal3-induced disruption is mediated by the gal3-carbohydrate-recognition domain and prevented by the gal3 inhibitor TD139, which also prevents AB42-induced degradation of gamma oscillations. Furthermore, we demonstrate that 5xFAD mice lacking gal3 (5xFAD-Gal3KO) exhibit WT-like gamma network dynamics.

Conclusions: We report for the first time that gal3 impairs cognition-relevant neuronal network dynamics by spike-phase uncoupling of FSN inducing a network performance collapse. Moreover, our findings suggest gal3 inhibition as a potential therapeutic target to counteract the neuronal network instability typical of $A D$ and other neurological disorders encompassing neuroinflammation and cognitive decline.

\section{Background}

Neuronal rhythmic electrical activity in the gamma-frequency band $(30-80 \mathrm{~Hz}$; gamma oscillations) underlies cognitive processes such as memory storage and recall, and plays a role in sensory perception [1-5]. This cognition-relevant brain rhythm emerges from the finely balanced and cooperative activity of particular neuronal populations within neuronal networks. As such, gamma oscillations depend on recurrent activity between excitatory pyramidal cells (PCs) [6] and the pacing of their activity by fast spiking perisomatic GABAergic interneurons that express parvalbumin (FSN) [7-9]. Thus, synchronized interplay between PCs and FSN generates and depends on the finely-patterned fluctuations of excitatory and inhibitory currents that give rise to oscillations of the local field potential $[10,11]$. The gamma rhythm is prominent in the hippocampus where its disruption has been reported to be linked to Alzheimer's disease (AD) [12-15].

$A D$ is a neurodegenerative disease characterized by diverse cellular and molecular pathological events that progressively lead to cognitive impairment. Such pathological events mainly, but not exclusively, comprise abnormal extracellular accumulation of beta-amyloid peptide (Aß42) [16-18], depositions of abnormal intraneuronal phosphorylated tau-tangles [19,20], defective synaptic transmission, and synaptic and neuronal loss, leading to marked neuronal network instability and deficient information processing [21]. There is growing evidence that in $A D$ synaptic failure and impaired network performance far precede $A \beta$ plaque deposition and clinical expression of cognitive decline [22-24]. 
Interestingly, diverse studies suggest that neuroinflammation, and specifically myeloid cells, play a major role in the initiation and progression of AD [25-29] with negative impact on brain circuit functioning [30, 31]. Recently, we found galectin-3 (gal3) to be a central regulator of the microglial immune response in Parkinson's disease $[32,33]$ and in prolonging sustained microglial activation in AD [26]. As such, microglia, the resident phagocytic immune myeloid cells of the Central Nervous System (CNS) are a recognized part of the cellular phase of AD [34].

Microglial activation has been reported to strongly correlate with amyloid deposition in patients with mild cognitive impairment (MCl) [35] and activated microglia have been found surrounding amyloid plaques [26, 36, 37] and neurofibrillary tangles [35, 38, 39], probably in an attempt to phagocytose them. However, their activation in AD becomes persistent and, eventually, ineffective [40,41]. Microglia-driven inflammatory responses include gal3 release, which has emerged as one of the foremost molecules in brain innate immunity associated with neurodegeneration $[26,42]$.

Gal3 has been found to act as an endogenous paracrine/autocrine toll-like receptor 4 (TLR4) ligand as well as the triggering receptor expressed on myeloid cells 2 (TREM2), binding through its carbohydrate recognition domain (CRD) [26, 32]. Moreover, gal3 has been found significantly upregulated in human AD patients compared to age-matched healthy controls [26]. Notably, it has been observed that gal3 inhibition decreases the inflammatory response of microglial cells treated with fibrillar $A \beta$. Moreover, a significant $A \beta$ plaque reduction and improvement of cognitive performance has been observed in 5xFAD mice lacking gal3. Also, gal3 is capable of binding TREM2, a central microglia receptor important in AD and essential for a functional microglial response. However, possible functional involvement of gal3 in the disruption of cognition-relevant neuronal network oscillations remains unknown.

Here, we set out to perform in vitro recordings of gamma oscillations in hippocampal area CA3 of WT and 5xFAD mouse brain slices. Gamma oscillations were induced by applying $100 \mathrm{nM}$ kainic acid (KA) either in an interface- or submerged-type recording chambers. Concomitantly to local field potential (LFP) recordings, we performed whole-cell patch-clamp recordings of FSN and PCs with emphasis on FSN activity. Our findings show the functional implications of gal3 signaling for neuronal network performance: It induces a functional collapse of neuronal network performance depending on gal3 concentration and activity state of the network. Moreover, gal3 inhibition prevents gal3-induced functional impairments and gal3 deletion counteracts disruption of gamma oscillations in the 5xFAD mice model at 6 months of age. Thus, our findings suggest removal or inhibition of gal3 as a potential therapeutic target to counteract the neuronal network instability typical of $A D$ and other neurological disorders encompassing neuroinflammation and cognitive decline.

\section{Material And Methods}

\section{Animals}

Experiments were performed in accordance with the ethical permit granted by Norra Stockholm's Djurförsöksetiska Nämnd (dnr N45/13) to AF and Malmö-Lund Ethical Committee on Animal Testing in Sweden (Dnr 5.8.18-01107/2018) to TD. For electrophysiological studies in WT brain slices, C57/BL6 male mice supplied from Charles River (Germany), were used at postnatal days 18 - 28. 5xFAD transgenic mice were acquired originally from Jackson Laboratories and crossbred at Lund University Animal Facility and were 
provided by TD and SB for electrophysiological studies. Gal3KO mice were provided by Dr. K. Sävman from Gothenburg University and crossbred at Lund's animal facility in homozygosis. 5xFAD-Gal3KO mice were generated by crossing heterozygous 5xFAD male mice with homozygous Gal3KO females to get 5xFAD ${ }^{+/-}$$\mathrm{Gal}^{+/-}$mice. Subsequent crossings between animals expressing this genotype, allowed for the generation of 5xFAD-Gal3KO mice. Ear biopsies were collected at the time of weaning at PD30. Mice were group-housed (4-5 animals/cage) in the Innovive disposable individually ventilated cage system (San Diego, USA) with water, food and nesting material provided ad libitum and following a $12 \mathrm{~h}$ light/dark cycle. Either 5xFAD, 5xFAD-Gal3KO, Gal3KO and WT mice were used at 6 months old.

\section{Genotyping}

Mice were genotyped for WT, 5xFAD or Gal3 by PCR as we have done previously (Boza-Serrano et al., 2019). Briefly, DNA from ear punches was extracted using the extraction kit (Extract-N-Amp ${ }^{\mathrm{TM}}$, Sigma-Aldrich) and amplified for PCR using the 2X PCR Bio HS Taq Mix Red enzyme (PCR Biosystems), with the following primers (5'-3'): For 5xFAD mice: App (Forward AGGACTGACCACTCGACCAG; Reverse CGGGGGTCTAGTTCTGCAT); Psn 1 (Forward AATAGAGAACGGCAGGAGCA; Reverse GCCATGAGGGCACTAATCAT; WT App (Forward CTAGGCCACAGAATTGAAAGATCT; Reverse GTAGGTGGAAATTCTAGCATCATCC). For Gal3KO mice: Gal3 (Forward: CACGAACGTCTTTTGCTCTCTGG); Ga/3//- Reverse: GCTTTTCTGGATTCATCGACTGTGG; $\mathrm{Gal}^{+/+}$ Reverse: TGAAATACTTACCGAAAAGCTGTCTGC.

\section{RNA extraction and RT-qPCR analysis}

Total RNA from frozen brain sections was extracted using TRI-reagent protocol (Sigma-Aldrich) following the manufacturer's instructions. RNA concentrations were measured using NanoDrop (2000C, Thermo), and $1 \mu \mathrm{g}$ of total RNA was converted to $\mathrm{CDNA}$ using iScript ${ }^{\mathrm{TM}}$ CDNA synthesis kit (BioRad). Real-time RT-qPCR was performed with SensiFAST ${ }^{\mathrm{TM}}$ SYBR No-ROX kit (Bioline) and $0.4 \mu \mathrm{M}$ of the following primer sequences $\left(5^{\prime}-3^{\prime}\right)$ : Trem2 (Forward: GTTTCTTGCAGCCAGCATCC; Reverse: GGGTCCAGTGAGGATCTGAAG); TIr4 (Forward: GGCATCATCTTCATTGTCC; Reverse: TCGAGGCTTTTCCATCCAA); Clec7a (Forward: CTGGTATGGAAGTAAGAGACACTGC; Reverse: CGGTGAGACGATGTTTGGC); Cx3cr1 (Forward: CAGCATCGACCGGTACCTT; Reverse: GCTGCACTGTCCGGTTGTT); Gfap (Forward: TCCTGGAACAGCAAAACAAG; Reverse: CAGCCTCAGGTTGGTTTCAT). Amplification was done in the CFX96 $6^{\text {TM }}$ Real-Time System-C $1000^{\mathrm{TM}}$ Thermal Cycler (BioRad) at $95^{\circ} \mathrm{C}$ for $2 \mathrm{~min}$, followed by 40 cycles at $95^{\circ} \mathrm{C}$ for $5 \mathrm{~s}$ and $60^{\circ} \mathrm{C}$ for $15 \mathrm{~s}$. Relative gene expression was represented as $\triangle \mathrm{Ct}$, normalized to Gapdh (Forward: ACCCAGAAGACTGTGGATGG; Reverse: ACACATTGGGGGTAGGAACA) mRNA levels.

\section{Hippocampal slice preparation}

Hippocampal slices were obtained as previously described [43-45]. In brief, after mouse sacrifice, the brain was quickly dissected out and placed in ice-cold artificial cerebrospinal fluid (ACSF) slightly modified for dissection, containing (in mM): $80 \mathrm{NaCl}, 24 \mathrm{NaHCO}_{3}, 25$ glucose, $1.25 \mathrm{NaH}_{2} \mathrm{PO}_{4}, 1$ ascorbic acid, 3 Na-pyruvate, $2.5 \mathrm{KCl}, 4$ $\mathrm{MgCl}_{2}, 0.5 \mathrm{CaCl}_{2}, 75$ sucrose and continuously bubbled with carbogen (95\% 02 and $5 \% \mathrm{CO} 2$ ). Horizontal hippocampal sections (350 $\mu \mathrm{m}$ thick) were obtained from both hemispheres with a Leica VT1200S vibratome (Leica Microsystems). After slicing, hippocampal slices were collected and allowed to recover in an interface recovery/holding chamber containing standard recording ACSF (in mM): $124 \mathrm{NaCl}, 30 \mathrm{NaHCO}_{3}, 10$ glucose, 
$1.25 \mathrm{NaH}_{2} \mathrm{PO}_{4}, 3.5 \mathrm{KCl}, 1.5 \mathrm{MgCl}_{2}, 1.5 \mathrm{CaCl}_{2}$. The holding chamber was continuously supplied with humidified carbogen gas $\left(5 \% \mathrm{CO}_{2}, 95 \% \mathrm{O}_{2}\right)$, held at $37^{\circ} \mathrm{C}$ during slicing and then allowed to cool down to room temperature for at least $1 \mathrm{hr}$ prior to start of any experiment.

\section{Electrophysiological recordings}

LFP recordings were performed either in interface- or submerged-type recording chambers in the hippocampal CA3 area with borosilicate glass microelectrodes filled with standard ACSF and placed in the stratum pyramidale. For LFP recordings in an interface configuration, slices were continuously supplied with aerated ACSF at a rate of $4.5 \mathrm{ml} / \mathrm{min}$ at $34^{\circ} \mathrm{C}$ and the chamber was continuously supplied with humidified carbogen gas. Single-cell recordings were carried out either concomitantly with LFP or on their own in a submerged recording chamber continuously supplied with aerated ACSF at a perfusion rate of $3 \mathrm{ml} / \mathrm{min}$ at $34^{\circ} \mathrm{C}$.

Depending on the configuration of patch-recordings (voltage- or current-clamp), different intracellular solutions were used. Action potentials (AP) and excitatory postsynaptic currents (EPSCs) from PCs and FSN were recorded in whole-cell mode with an internal recording solution for current-clamp configuration containing (in $\mathrm{mM}$ ): $122.5 \mathrm{~K}^{+}$-gluconate, $8 \mathrm{KCl}, 2 \mathrm{Mg}^{2+}$ ATP, $0.3 \mathrm{Na}^{+} \mathrm{GTP}, 10$ HEPES, $0.2 \mathrm{EGTA}, 2 \mathrm{MgCl}$. An internal recording solution for voltage-clamp containing (in mM) 140 CsMetSO $_{4}, 2 \mathrm{Mg}^{2+}$ ATP, $0.3 \mathrm{Na}^{+} \mathrm{GTP}, 10$ HEPES, and 0.6 EGTA was used for PC- inhibitory postsynaptic currents (IPSC) recordings. For each internal solution pH was set to 7.2-7.3 and osmolarity to 270-280 mosmol/I. PC- and FSN-EPSCs, and PC-IPSCs were recorded in voltageclamp configuration holding the membrane potential at $-70 \mathrm{mV}$ or $0 \mathrm{mV}$, respectively. FSN- membrane potential (Em) and firing threshold were recorded in current-clamp configuration at resting membrane potential in gapfree for Em, or by applying current steps of $10 \mathrm{pA}$ starting at $-70 \mathrm{mV}$ holding membrane potential for firing threshold.

PCs and FSN were visually identified under an upright microscope using IR-DIC microscopy (Axioskop, Carl Zeis AG, Göttingen, Germany) based on their morphology and location in the hippocampal str. pyramidale and str. radiatum, respectively. PCs and the interneuron subtype (FSN) were further confirmed as previously described by applying different stimulation protocols to verify the cellular population type by their unique electrophysiological characteristics [24, 44, 46, 47]. For recordings of PC or FSN in whole-cell configuration access resistance was monitored throughout the experiment. Cells were excluded from the study if more than $20 \%$ of change was observed.

Gamma oscillations were induced by applying 100 nM kainic acid (KA, Tocris) $[43,44]$ and allowed to stabilize prior to recording of any cellular/LFP parameter. LFP recordings in the interface-type recording chambers were acquired with a 4-channel M102 amplifier (University of Cologne, Germany). Data was sampled at $10 \mathrm{kHz}$, conditioned using a HumBug $50 \mathrm{~Hz}$ noise eliminator (Quest Scientific), low-pass filtered at $1 \mathrm{kHz}$, digitized using a Digidata 1440A (Molecular Devices, CA, USA) and stored using pCLAMP 9.6 software (Molecular Devices, CA, USA). LFP and patch-clamp recordings in submerged-type recording chamber were acquired with a patch-clamp amplifier (Multiclamp 700B) using pCLAMP 10.4 software (Molecular Devices, CA, USA). LFPs recorded in the submerged-type recording chamber were also conditioned using a HumBug $50 \mathrm{~Hz}$ noise eliminator (Quest Scientific). All signals recorded in submerged configuration were low-pass filtered at $1 \mathrm{kHz}$, acquired at $5 \mathrm{kHz}$, digitized and stored using Digidata 1322A and pCLAMP 10.4 software (Molecular Devices, CA, USA). 


\section{Data analysis}

For power spectral density plots, fast Fourier transformations (segment length 8192 points) were calculated from 60 s-long LFP recordings using Axograph X (Kagi, Berkeley, CA, USA). Gamma oscillation power was calculated by integrating power spectral density between 20 and $80 \mathrm{~Hz}$ using KaleidaGraph. Peak frequency of gamma oscillations was obtained using Axograph X. For further analysis of LFP recordings, the signals were filtered in both directions using a band-pass Butterworth filter set to $20-50 \mathrm{~Hz}$ for autocorrelation analysis of gamma oscillations. Normalized autocorrelations were performed using Matlab custom-written routines. The coefficient of rhythmicity $(\mathrm{Cr})$ was calculated from the autocorrelograms as a measure of the quality of gamma oscillations $[43,48]$ or EPSCs and IPSCs rhythmicity [48]. It was defined as $\mathrm{Cr}=(\alpha-\beta) /(\alpha+\beta)$ where $(1+\alpha)$ and $(1$ $+\beta)$ corrections were applied. The value of a corresponds to the value of the height of the second peak and $\beta$ to the first trough in the autocorrelogram counting the first peak at zero lag. $\mathrm{Cr}$ ranges between 0 and 1 with higher coefficient values denoting more rhythmic activity and thus better rhythm quality. Only recordings with $\mathrm{Cr} \geq$ 0.01 were included in the study.

FSN- and PC-spike-phase coupling with corresponding gamma-LFP (60 s-long) were analyzed using a custommade Matlab routine as previously described [43, 44, 48]. LFP traces were filtered using a band-pass Butterworth filter (20-40 Hz, both directions) and AP were detected using an amplitude threshold set to $30-50 \%$ of the total AP amplitude. The instantaneous phase-angle of gamma oscillations for each AP was determined using a Hilbert transform. Firing window was analyzed by distributing phase-angles of all AP and gamma oscillation-phases (in radians) in polar-plots with the peak of the oscillation cycle corresponding to $0 \pi$ and the trough to $\pi$ in the polar plots. For each AP, a vector of length $=1$ was assigned at the angle corresponding to the phase of the LFP. An averaged resultant phase-density vector was used to describe the preferred phase of firing (phase-angle) and how recurrent the firing in that angle was (vector length). Thus, a larger vector denotes a stronger spike-phase coupling/more synchronized AP firing relative to the network gamma oscillation. Vector length is shown normalized by the total number of AP for each cell recorded and the summary is plotted for each condition. All firing distributions were assessed for uniformity setting the inclusion criteria at $p<0.05$ when performing a Rayleigh's test.

EPSCs and IPSCs were detected off-line using a custom-made template in Clampfit 10.4 software including no less than 20 averaged representative events. EPSC and IPSC parameters (charge transfer, event amplitude and inter-event-interval (IEI) distributions) were performed and analyzed using GraphPad Prism (GraphPad Software, USA) with the results representing average values taken over $60 \mathrm{~s}$ periods. For the time domain analysis (cross-correlation) the same $60 \mathrm{~s}$ segment of EPSCs/IPSCs and their corresponding LFP were filtered (Butterworth, 20-40 Hz). Normalized cross-correlation between EPSCs/IPSCs and the LFP (XC(EPSCs/IPSCs,LFP)) was performed with a Matlab custom-written routine. The cross-correlation analysis displayed several peaks with periodic fluctuations evidencing the oscillatory nature of the signals. The magnitude and lag of the central negative peak from the XC function were used to describe the similarity and phase-shift of both signals, respectively $[48,49]$.

\section{Drugs and chemicals}

All chemical compounds used in intracellular and extracellular solutions were obtained from Sigma-Aldrich Sweden AB (Stockholm, Sweden). KA was dissolved in miliQ water. Human gal3 protein was produced by the 
protein production unit LP3 at Lund University. Briefly, the gal3 protein was produced in strain Escherichia coli TUNER(DE3) / pET3c-hum-gal3 grown in LB medium, $18^{\circ} \mathrm{C}, 250 \mathrm{rpm}$ with $1 \mathrm{mM}$ IPTG overnight. After cell lysis and ultracentrifugation, gal3 was purified on a $20 \mathrm{ml}$ lactocyl-sepharose column. Peak fractions containing gal3 were pooled and dialyzed against PBS, pH 7.4. Human gal3 was analyzed on a Criterion TGX AnykD precast SDS-PAGE gel (Bio-Rad) stained with Bio-Safe Coomassie (Bio-Rad). The purity was estimated to $>95 \%$. The inhibitor TD139 (1,1'-sulfanediyl-bis-3-deoxy-3-4-3-fluorophenyl-1 H-1,2,3-triazol-1-yl- $\beta$-d-galactopyranoside) was acquired from Chemtronica (Stockholm, Sweden) and dissolved in DMSO to a final dilution $\leq 0.001 \%$ of DMSO when applied to brain slices.

\section{Statistical analysis}

All statistical analysis was performed using GraphPad Prism 8.0 either in absolute values or normalized data (to $5 \mathrm{~min}$ of stable baseline) when appropriate. To minimize the variation between slices, a paired statistical design was used when compounds were washed-in sequentially and unpaired when treatments were performed independently of each other. Two- or one-sided designs were used as appropriate. Prior to statistical analysis all data were subjected to detection of outliers that were removed using the ROUT method. This was followed by tests for normality distribution and variance similarity between groups. Wilcoxon's signed rank sum test was used when reporting statistical differences between control conditions and compound effects in the nonparametrically distributed data. A paired Student's t test was used when the data were distributed normally. For multiple comparisons, ordinary one-way ANOVA followed by Holm-Sidak's multiple comparisons test or KruskalWallis test followed by Dunn's multiple comparisons were carried out depending on the parametric or nonparametric nature of the data, respectively. Results are reported as mean \pm SEM and significance levels were set as follows: ${ }^{*} p<0.05, * * p<0.01, * * * p<0.005, * * * * p<0.0001$.

\section{Results}

\section{Gal3 induces degradation of gamma oscillations via its carbohydrate recognition-domain}

We first evaluated the effect of gal3 on gamma oscillation power and rhythmicity. For this purpose, hippocampal slices of WT mice were incubated for 15 min with $1 \mu \mathrm{M}$ gal3 and transferred to an interface-type recording chamber. Gamma oscillations were elicited with $100 \mathrm{nM} \mathrm{KA}$ and allowed to stabilize for 30 min following which gamma-local field potential (gamma-LFP) was recorded and analyzed [43, 45]. Incubation with gal3 prior to gamma oscillation induction resulted in a drastic decrease of gamma oscillation power (Fig. 1C, Supplementary Table 1, Additional file 1) and rhythmicity (Fig. 1D, Supplementary Table 2, Additional file 1), as observed in the $\mathrm{Cr}$ analysis used as an indicator of gamma oscillation quality (see Methods). Co-incubation of slices with gal3 and 1 or $3 \mu \mathrm{M}$ of gal3 inhibitor TD139 prior to gamma oscillation induction did not prevent gal3-induced decrease of gamma oscillation power while $10 \mu \mathrm{M}$ TD139 conferred an efficient protection (Fig. 1C, Supplementary Table 1, Additional file 1). Treatment with $10 \mu \mathrm{M}$ TD139 alone did not exert additional changes compared to control gamma power (Fig. 1C, Supplementary Table 1, Additional file 1). Interestingly, Cr analysis revealed that 1 and $3 \mu \mathrm{M}$ TD139 partially prevented gal3-induced disruption of gamma rhythmicity and $10 \mu \mathrm{M}$ TD139 fully counteracted this gal3-mediated loss of gamma quality (Fig. 1D, Supplementary Table 2 , Additional file 1). As for the power analysis above incubation of the neuronal network with $10 \mu \mathrm{M}$ TD139 alone did not change gamma oscillation rhythmicity (Fig. 1D, Supplementary Table 2, Additional file 1). 
Prominent cellular actions of gal3 have been reported to be mediated by its characteristic carbohydrate recognition-domain (CRD) [26,32]. Thus, we investigated whether the deleterious effects of gal3 on network oscillations were driven by its CRD. For this purpose, hippocampal slices of WT mice were incubated for 15 min with $1 \mu \mathrm{M}$ gal3-isolated CRD as well as with $1 \mu \mathrm{M}$ R186S-Gal3 and then transferred to an interface-type recording chamber. R186S-Gal3 is a mutated version of gal3 with reduced affinity for many glycoproteins and for the disaccharide LacNAc, which is the most common minimal galectin-binding moiety in glycoproteins [50, 51]. Recordings performed $30 \mathrm{~min}$ after the induction of gamma oscillations revealed that the effects of gal3CRD parallel those of intact gal3: Decrease of gamma power (Fig. 1E) and impairment of gamma oscillation rhythmicity (Fig. 1F). Notably, R186S-Gal3 did not affect either gamma power (Fig. 1E) or rhythmicity (Fig. 1F). Thus, gal3 induces a drastic degradation of gamma oscillations in the hippocampal network through its CRD and this effect is prevented with highest efficacy by $10 \mu \mathrm{M}$ TD139.

\section{Gal3 disrupts action potential phase-lock of fast-spiking interneurons to gamma oscillations in a concentration- and exposure timedependent manner}

We evaluated the effect of gal3 on fast-spiking interneuron (FSN) phase-lock to ongoing gamma oscillations. To this end, we performed concomitant recordings of LFP and whole-cell patch-clamp recordings of FSN activity in a submerged-type recording chamber during the activated network state (gamma oscillations). Thirty minutes after gamma oscillation induction, 5 min of control gamma and FSN firing were recorded and subsequently gal3 was bath-applied. Application of $1 \mu \mathrm{M}$ gal3 for $30 \mathrm{~min}$ did not induce a decrease of gamma oscillation power (Fig. 2A, B) indicating that its effect may depend on the activation state of the network. Likewise, FSN firing phase-lock and AP firing rate were not affected by the application of $1 \mu \mathrm{M}$ gal 3 to the activated network (Supplementary Figure 1, Additional file 2).

Using the same approach of submerged-type recording chamber and gal3 application to the activated network we increased gal3 concentration and the time of application. $2 \mu \mathrm{M}$ gal3 gradually decreased gamma oscillation power starting $15 \mathrm{~min}$ after application and reaching a significant stable reduction at $40 \mathrm{~min}$ after wash-in (Fig. 2C, D). Concomitantly, FSN-gamma phase-lock was severely disrupted (Fig. 2E - I) evidenced by a significant decrease of the resultant vector length of the AP firing distribution within gamma phases (Fig. 2E, $\mathrm{G})$. The preferred gamma phase-angle was not affected (Fig. 2H) whereas phase-lock impairment was accompanied by a drastic decrease of FSN firing rate (Fig. 2E, F, I).

During ongoing gamma oscillations, FSN pace the activity of pyramidal cells (PCs) $[7-9,46]$. Thus, any alterations of FSN activity within the network could affect PC activity during ongoing gamma oscillations. To further address this possible causal link to PCs we performed the same experiment used for assessing FSN (Fig. 2E-I) in PCs. Similar to the FSN experiment, 40 min application of $2 \mu \mathrm{M}$ gal3 during ongoing gamma oscillations resulted in a drastic desynchronization of PC-AP firing (Fig. 2J-N). This was evidenced by a widening of the firing window, which resulted in a shorter vector length (Fig. $2 \mathrm{~J}, \mathrm{~L}$ ) that was accompanied by a drastic decrease of AP firing rate (Fig. 2J, K, N). The preferred phase-angle of AP firing was not affected by gal3 wash-in (Fig. 2M).

\section{Gal3 impairs inhibitory and excitatory synaptic transmission during ongoing gamma oscillations}


Proper coordination of excitatory and inhibitory synaptic activities within neuronal networks is crucial for circuit entrainment and emergence of brain rhythms $[9,11,46,52,53]$. Here, we have observed that gal 3 impairs the firing coordination relative to gamma oscillations of crucial neuronal populations such as inhibitory GABAergic FSN and excitatory glutamatergic PCs. We proceeded to investigate the effect of gal3 on the excitatory and inhibitory synaptic inputs onto PCs as well as excitatory drive onto FSN. For this purpose, we recorded excitatory and inhibitory postsynaptic currents (EPSCs and IPSCs, respectively) from PCs (see methods) 40 min after $2 \mu \mathrm{M}$ gal3 application during ongoing gamma oscillations (Fig. 3).

Analysis of EPSC charge transfer showed that gal3 significantly decreased the overall excitatory input onto PCs (Fig. 3A), which is accompanied by a significant decrease of EPSC frequency (Fig. 3C). At the same time gal3 causes a shift of the EPSC amplitude distribution towards smaller components (Fig. 3D) while the mean amplitude remains unaffected (Fig. 3B). Additionally, gal3 severely impairs both the EPSC rhythmicity within the gamma frequency-band and the PC-EPSC to gamma-LFP phase relationship (Fig. 3I-K).

Interestingly, inhibitory drive onto PCs also showed a decrease $40 \mathrm{~min}$ after gal3 application. This was evidenced by a significant decrease of the overall IPSC charge transfer (Fig. 3E) caused by a decrease of IPSC amplitude (Fig. 3F). In contrast, IPSC frequency remained unchanged after gal3 wash-in (Fig. 3G). Gal3 also shifted the IPSC amplitude distribution decreasing larger amplitude components while inducing an increase of smaller amplitude components (Fig. 3H). Similarly to EPSCs, gal3 severely impaired IPSC rhythmicity and, notably, the PC-IPSC to gamma-LFP phase relationship was drastically weakened (Fig. 3L-N).

Gal3 also decreased the overall excitatory input onto FSN as evidenced by a significant decrease of EPSC charge transfer (Fig. 30) that was accompanied by a decrease of EPSC amplitude (Fig. 3P) and increased occurrence of small amplitude components (Fig. 3R). However, overall mean FSN-EPSC frequency was not affected (Fig. 3Q). Notably, gal3 also disrupted EPSC rhythmicity in FSN (Supplementary Figure 2A, Additional file 2) as well as the relationship between EPSCs and LFP within the gamma frequency-band (Supplementary Figure 2B, Additional file 2). These findings show that gal3 dramatically impairs overall excitatory and inhibitory inputs onto PCs. This is accompanied by a loss of excitation and inhibition rhythmicity, and an impairment of the relationship between postsynaptic currents and the gamma rhythm, a finding that shares commonalities with the observed gal3-induced impairment of excitatory drive onto FSN.

\section{Neuronal mechanisms underlying the activity-dependence of gal3-induced impairment of functional network dynamics}

Here we have observed significant reductions of gamma power and rhythmicity when slices are exposed to gal3 $(1 \mu \mathrm{M})$ prior to gamma induction (see Fig. 1). However, when the network is under stable entrainment into the gamma rhythm ( 30 min after gamma induction by $100 \mathrm{nM} \mathrm{KA}$ ), an increased concentration of gal 3 is needed to observe comparable disruption (see Fig. 2). To investigate the effects of gal3 on FSN basal activity and its possible consequences for the network function during gamma oscillations, we set out to perform recordings of FSN activity concomitantly with LFP first in the basal state and then during subsequent gamma oscillation induction in area CA3 of hippocampal WT mouse slices (Fig. 4A). Gal3 or gal3 plus gal3 inhibitor (TD139) were applied for 15 min prior to gamma oscillation induction and remained in the bath solution throughout the subsequent $30 \mathrm{~min}$ of rhythm establishment and stabilization. Basal state recordings also 
served as control to ensure that neither the whole-cell patch-clamp configuration nor the bath ACSF induced functional deviations during the total 45 min-long recordings (Fig. 4A).

Recordings of FSN membrane potential $(\mathrm{Em})$ in quiescent state revealed that gal3 hyperpolarizes FSN Em 15 min after bath application (Fig. 4B, D). Co-application of gal3 and TD139 prevents this gradual hyperpolarization (Fig. 4B, E), which is also absent in the control condition (ACSF, Fig. 4B, C). In parallel to the observed hyperpolarization of FSN Em, gal3 induced an increase of the FSN AP firing threshold (Supplementary Figure 3B, Additional file 2) which, again, was prevented by co-application of TD139 (Supplementary Figure 3C, Additional file 2) and absent in the control condition (ACSF, Supplementary Figure 3A, Additional file 2). Interestingly, we found that some FSN were spontaneously firing APs at basal Em without significant changes of their firing rate during the 15 min of quiescent state recording (Fig. 4E and Supplementary Figure 3C, Additional file 2).

We also observed a drastic decrease of the firing rate at basal state following gal3 wash-in (Fig. 4E, inset and Supplementary Figure 3E, Additional file 2), probably due to the gal3-induced hyperpolarization of FSN Em and the increase of the firing threshold. The decrease of the firing rate was prevented by the co-application of TD139 (Fig. 4E and Supplementary Figure 3F, Additional file 2). At the network level, gal3 provoked a decrease of EPSCs frequency onto FSNs that was prevented by co-applying TD139 (Supplementary Figure 4D-F, Additional file 2). Thus, our findings indicate that $1 \mu \mathrm{M}$ gal3 in quiescent state causes hypoactivity in FSN prior to gamma oscillation induction, which then overrides that hypoactivity to a limited extent. This is evident in the fact that concomitant LFP recordings revealed that the neuronal network fails to generate gamma oscillation power similar to that seen in control conditions (Fig. 4F, G). Such gal3-induced impairment of rhythmic network activity is counteracted by the presence of TD139 (Fig. 4F, G). Additionally, gamma oscillations induced in slices previously exposed to gal3, showed lower peak frequency compared to gamma oscillations recorded in control conditions or in the presence of the gal3 inhibitor (Fig. 4F, H).

Treating slices with gal3 led to a drastic decrease of FSN AP firing rate during ongoing gamma oscillations (Fig. 4F, I) $[44,46,48,54]$. By contrast, FSN recorded under gal3 inhibition displayed similar firing rates to FSN recorded in control conditions (Fig. 4F, I). Notably, gal3 significantly prevented FSN spike-phase locking to gamma LFP, evidenced by a drastic decrease of the resultant vector length (Fig. 4J). This was accompanied by an increase of the ratio of FSN that did not show patterned activity relative to the ongoing gamma rhythm (Fig. 4K). Gal3 also led to decreased EPSCs frequency and disrupted EPSCs rhythmicity onto FSN (Supplementary Figure 5B and D, Additional file 2). These functional deviations were prevented in the presence of TD139. In addition, analysis of FSN Em in the activated network state (gamma oscillations) revealed that gal3-driven decrease of FSN firing rate during gamma oscillations was not caused by the previous hyperpolarization observed in the quiescent state. KA-induced depolarization during gamma oscillation induction resulted in a similar FSN Em in the three studied conditions: (1) control, (2) gal3 and (3) gal3 + TD139 (Fig. 4N).

To investigate a possible effect of gal3 in some of the gal3 activation/responses-associated genes related to microglial AD-phenotype, we assessed the expression of Trem2, TIr4, Clec7a, and Cx3cr1 transcripts in some of the recorded slices. Notably neither Trem2, Clec7a nor T/r4 changed their expression in slices treated with gal3 prior to gamma oscillation induction (Fig. S6). Conversely, there was a decrease of the $\Delta \mathrm{Ct}$ of the astrocyte marker Gfap in slices treated with gal3 in the presence of the inhibitor TD139. $\Delta \mathrm{Ct}$ of the chemokine receptor 
Cx $3 \operatorname{cr} 1$ revealed that $\mathrm{KA}$ induces a decrease of this parameter while neither gal3 nor gal3 upon inhibition increased the $\Delta \mathrm{Ct}$ (Supplementary Figure 6, Additional file 2).

\section{Interference with gal3-signaling prevents the disruption of gamma oscillations in two different AD models}

Acute application of physiological concentrations of $A \beta 42(50 \mathrm{nM})$ to hippocampal slices causes a dramatic and rapid impairment of gamma oscillations [43-45, 47, 55, 56]. Also, cognition-relevant brain rhythms have been reported to be disturbed in several AD mouse models [24,57-61]. We investigated whether inhibition of gal3 signaling in two AD-related mouse models is able to prevent the disruption of gamma oscillations, which is linked to the cognitive impairment in $A D$.

Firstly, we performed recordings of gamma oscillations in area CA3 of WT mice hippocampal slices previously incubated with A $A 42$ or A 42 + TD139 ("acute A $\beta$ mouse model"). Recordings were performed in the same configuration as for the experiments in Fig. 1 (interface-type recording chamber, gamma oscillations induced after 15 min of incubation with $50 \mathrm{nM} \mathrm{A \beta 42} \mathrm{or} 50 \mathrm{nM} \mathrm{A \beta 42} \mathrm{+} 10 \mu \mathrm{M}$ TD139 and recorded 30 min after rhythm induction). In slices pre-incubated with $A \beta 42$, gamma oscillation power and rhythmicity were significantly decreased, in accordance with previous reports $[43,45,48,55,56]$. Surprisingly, co-incubation with TD139 fully prevented this $A \beta 42$-induced functional impairment (Fig. 5A, C, D).

Secondly, we tested whether gamma oscillations were impaired in a familial AD mouse model also ("chronic 5xFAD mouse model"), similarly to what we had demonstrated recently in another chronic AD model $\left(A p p^{N L-G-F} ;[24]\right)$, and, if so, whether gal3 antagonism would then also effect a rescue of network performance. 5xFAD mice exhibit a dramatically accelerated $A \beta 42$ production with visible $A \beta$ deposits starting as early as 2 months-of-age (A $\beta$ plaque surrounded by gal3+ microglia) [26] and progressive cognitive deficits as early as 45 months-of-age $[62,63]$. Notably, gal3 deficiency reduces $A \beta$ plaque burden and plaque size, and improves cognitive performance in 5xFAD-Gal3KO mice at 6 months-of-age [26].

In the present study, we observed that the hippocampal CA3 network of 6 months-old 5xFAD mice exhibits a significant decrease of gamma oscillation power and a shift of the peak gamma frequency towards lower values. Notably, in age-matched 5xFAD-Gal3KO mice, gamma oscillation power and peak frequency were unaffected and at WT littermate control levels. Gal3 deletion did not cause further changes in both parameters (Fig. 5B, E, and F). Thus, gal3 parallels the toxic effects of $A \beta 42$ on gamma oscillations as previously reported $[43-45,48,55,56]$. This toxicity was prevented by gal3 inhibition (see Fig. 1) while gal3 deletion prevents functional impairment of the gamma network rhythm in the 5xFAD AD mouse model.

Taken together our results suggest that gal3 not only governs the microglial neuroinflammatory response as we previously demonstrated [26] but, as shown in this study, can directly be connected to AD-related aberrant synchronization in neuronal networks. Importantly, we can pharmacologically rescue this aberrant neuronal network behavior by intervening at the CRD site of gal3. This suggests gal3 as a potential therapeutic target effective not only against neurodegenerative processes, but also in rescuing normal neuronal signaling, cognition-relevant brain rhythms and so, presumably, having a positive effect on cognitive decline.

\section{Discussion}


Synchronization of fast neuronal activity between PCs and FSN within hippocampal CA3 circuits give rise to the emergence of periodic fluctuations of the LFP in the gamma frequency-band $(30-80 \mathrm{~Hz}$, gamma oscillations) (Fisahn et al., 1998). Such activities comprise phase-locked AP firing and rhythmic inhibitory and excitatory postsynaptic currents (IPSCs and EPSCs respectively), as shown in the current study. Particularly, FSN command the entrainment of PC activity during gamma oscillations and provide stability to the neuronal network performance. FSN are therefor a suitable target to counteract deficient cognitive performance $[8,9,15$, $24,46,47,53,60,64]$. The coordinated and cooperative activity within the network allows for the binding of neurons in neuronal ensembles that then process sensory information, thus supporting cognitive processes and memory formation [65]. Therefore, any functional deviation such as impairment of FSN activity, inhibitory and excitatory synaptic transmission, and consequent aberrant $\mathrm{PC}$ activity may negatively impact overall network performance with detrimental cognitive implications as observed in AD.

In this regard, we have previously observed that $A \beta 42$ induces synaptic failure as well as desynchronization of PC AP firing [43, 45], degradation of FSN spike phase-locking $[44,48]$ relative to gamma oscillations, and overall neuronal network impairment $[43,44,48,55,56,66]$. Interestingly, the current study reveals for the first time that acute gal 3 induces a functional network collapse that shares commonalities with the reported effects of $A \beta 42$ on neuronal networks dynamics [43-45, 48,67]. Moreover, a possible association of gal3 and Aß42 underlying detrimental microglia activation in $A D$ has been described recently [26]. Despite huge efforts towards counteracting the pathological events leading to progressive cognitive impairment in $A D$, disease-modifying approaches have failed or shown only modest advances (see Isla et al., 2021) [61]. One possible shortcoming of past therapeutic attempts is the fact that their focus has been on mere A 342 plaque removal [61], whereas growing evidence points to neuronal synchronization as well as neuroinflammation as critical targets to counteract the progression of AD [25-28]. Circuit entrainment into physiological brain rhythms on one hand side has gained prominence as a suitable and promising approach to counteract progressive neuronal network dysfunction and deficient cognitive performance in $\operatorname{AD}$ with focus on FSN activity $[44,58,60]$. On the other hand side, microglial gal3 has been proposed as a central regulator of the microglia-driven neuroinflammatory responses in $A D$ [26]. We found here that inhibition or deletion of gal3 counteracts all the functional deviations it induces by preserving normal neuronal network dynamics that are relevant for cognitive capabilities.

Gal3 belongs to a protein family with at least 15 members that have substantial sequence similarity in their CRD and binds to $\beta$-galactosides with variable affinities and specificities $[68,69]$. Gal3 has been found associated with microglial cells in close vicinity of AB42-containing plaques in AD patients as well as animal and cellular models [26]. However, its functional impact once released by activated microglia on neighboring neurons and synapses farther from $A \beta 42$ plaques should also be considered. Here, we have observed that preincubation of the hippocampal neuronal network with gal3 induces a drastic degradation of gamma oscillations mediated by its CRD. Interestingly, gal3 has been reported to bind to and activate different microglial receptors, which include TREM2 [26] and TLR4 [32]. Particularly, binding to TREM2 and TLR4 has been described to be mediated by gal3 CRD [26,32]. Accordingly, the consistent prevention of gal3-mediated disruption of neuronal and network function observed in the presence of the gal3 inhibitor TD139 reinforces our findings.

TD139 is a 3,3'-Bis-(4-aryltriazol-1-yl) thio-digalactoside gal3 inhibitor with high affinity for the gal3-CRD. In this study we tested whether the drastic disruption of gamma oscillations observed following exposure of the

Page $12 / 33$ 
hippocampal network to gal3 is accompanied by changes in the expression of genes related to microglial activity. We observed changes in the expression of very few genes, which could be ascribed to the relatively short exposure time (45 min total, see Fig. 5) that appears long enough to prevent an efficient induction of gamma oscillations but too short to trigger a significant change of gene expression. However, the overall quantification of the expression of the listed transcripts in the present study should just be taken as a descriptive clue and future experiments should be performed to further assess a wider range of microglia activation-related genes with longer gal2 exposure-times.

The significant electrophysiological changes to neurons and networks observed here appear to rely on a mechanism linked to the cell membrane (i.e., a particular microglial receptor), probably inducing an early microglial activation with further consequences if the application lasts longer as expected from the timedependence shown in Fig. 1, and as it may happen in vivo during sustained gal3 release. Again, the overall preventive effect of TD139 reinforces this notion. TD139 mainly acts extracellularly within the time of application employed here. The inhibitor does not reach the intracellular compartments unless incubation/wash-in lasts 24 hours or more [70].

Furthermore, we found that gal3 is less efficient in disrupting gamma oscillations if the network is already properly entrained into the gamma rhythm (see Fig. 2). However, if the network is treated with gal3 prior to FSN engagement into strong spike-phase coupling, gal3 prevents the proper establishment of a coordinated activity that leads to gamma oscillations of physiological relevance (see Fig. 4). In an AD scenario, it is tempting to hypothesize that at a certain point during very early pathology progression, cognition-relevant neuronal networks preserve their functionality due to homeostatic mechanisms (a large concentration of gal3 is needed to disrupt cellular and neuronal normal activities). As the pathology progresses, such homeostatic mechanisms become overwhelmed, perhaps even deleterious (i.e., microglia shifts to damage-associated microglia (DAM) phenotype (see Frere and Slutsky, 2018) [21]). Then, a progressively weakened neuronal network appears more susceptible to gal3 that could reach the neurons/synapses by diffusing throughout the brain parenchyma once secreted by the already activated microglia (a lesser gal3 concentration drives major dysfunction).

A plausible explanation for the drastic gal3-induced effects observed could be found in the complex purinergic signaling in the neuron-microglia crosstalk. It has been proposed that the initial increase of extracellular adenosine to levels far greater than reached in physiological conditions initially leads to a burst of adenosine receptor $1\left(A_{1} R\right)$-mediated inhibition, and the continuous massive overflow of extracellular adenosine then overcomes the restricted activation of $A_{2 A} R s$, which results in a predominant role of $A_{2 A}$ Rs in the development of neurodegeneration [71]. In line with this proposal a paramount impact of ATP/adenosine signaling on hippocampal circuitry function has been observed. At mossy fiber-CA3 synapses microglia-derived ATP differentially modulates synaptic transmission and short-term plasticity through activation of presynaptic P2X4 receptors and $A_{1} R$, respectively, with the latter following its extracellular conversion to adenosine [72].

Additionally, blockade of $A_{2 A}$ Rs prevents lipopolysaccharides-induced impairment of long-term potentiation in rat in vivo, by counteracting the shift of microglia towards a pro-inflammatory phenotype [73]. Notably, $A_{2 A} R$ is upregulated in APP/PS1 mice model [74] as well as in cortical areas [75] and the hippocampal formation of AD patients [76]. It has been previously found that adenosine inhibits KA-induced and spontaneous gamma oscillations, particularly via the activation of $A_{1} R$ [77]. Consistent with our hypothesis and results, it has been observed that endogenous ATP release drastically reduce PC AP firing rate as well as spike synchronization 
during KA-induced gamma oscillations in the CA3 area. Moreover, both KA- and acetylcholine-induced gamma oscillations are inhibited by ATP/adenosine receptor activation [78].

In this study we found a drastic decrease of both excitatory and inhibitory drive onto PCs as well as a decrease of excitatory input onto $F S N$. In this regard, hippocampal $A_{1}$ Rs are known to hyperpolarize $F S N$, reduce the excitability of PCs and interneurons, and also reduce neurotransmitter release $[79,80]$. Changes in neurotransmitter release are mostly associated with changes of frequency of synaptic events, here observed as a decrease of EPSCs onto PCs (see Fig. 3B) and FSN (see Supplementary Figure 5, Additional file 2) in the presence of gal3. Notably, gal3 reduced the occurrence of larger IPSC components in PCs probably as a reflection of the observed FSN impairment since the major perisomatic inhibition of PCs is driven by FSNs [8, 9]. The overall collapse of the network was also observed in the gal3-induced increased variability of the phase relation between both EPSCs and IPSCs with the corresponding LFP-gamma as well as the loss of the rhythmicity of the postsynaptic currents. This loss of rhythmicity of cellular electrical events likely accounts for the degradation of the network rhythm since the generation of gamma oscillations depends on balanced excitatory and inhibitory interplay [11]. However, due to the diverse evidence of purinergic signaling in the modulation of the operational capacity of the hippocampal circuitry, and commonalities found in our study regarding gal3-induced neuronal network collapse, a possible underlying mechanism involving ATP/adenosine receptor activation deserves further research without excluding an indirect involvement of astrocytes, nitric oxide and metabolic arrestment $[30,31,76,81]$ and a direct effect of gal3 on PCs and FSN.

Finally, there is mounting evidence suggesting that in AD synaptic and network failure starts long before the establishment of $A \beta 42$ depositions into solid plaques and manifestation of cognitive decline expression [21, 59, 82-84]. Interestingly, in the APP/PS1 mouse model associative long-term synaptic plasticity is impaired in CA3 $P C s$ at the early onset of $A D$-like features due to the postsynaptic activation of upregulated $A_{2 A} R$ [74]. Moreover, focal glial activation has been reported to precede amyloid plaque deposition in APP transgenic mice associated with a vicious cycle of APP proteolytic cleavage that gives rise to soluble and amyloidogenic immunostimulatory mediators [27]. Using a proteomic approach, it has been found that immune alterations in microglia in 5XFAD mice are active before plaque deposition [85].

Recently, the notions on direct involvement of microglia-released gal 3 and $A \beta$ cross-seeding agents in plaque formation has been validated $[26,86,87]$. Particularly, the 5xFAD mouse model lacking gal3 (5xFAD-Gal3KO) fails to develop prominent $A \beta$ plaques and cognitive impairment typical of the 5xFAD model at 6 months-of-age [26]. Here we found that the underlying functional reason explaining these previous reports could be the significant degradation of gamma oscillations (observed in the 5xFAD model at 6 months-of-age) that is absent in the age-matched 5xFAD mice lacking gal3 (see Fig. 5). 5xFAD mice also showed a significant slowing of gamma oscillation-frequency compared to WT control that was not evident in the age-matched 5xFAD-Gal3KO, which retained values similar to WT. Interestingly, slower gamma oscillations have been observed also in the CA3 area of organotypic hippocampal cultures under microglial priming with interferon gamma [88]. Inhibition of gal3 by co-application of TD139 prevents the decrease of both gamma oscillation power and frequency in the acute $A \beta$ application model (see Fig. 5).

\section{Conclusions}


In summary, here we report for the first time that gal3 - a proposed central microglial/neuroinflammatory regulator in $A D$ - causes degradation of cognition-relevant neuronal network dynamics and their underlying neuronal synchronization mechanisms. These impairments are mediated by the gal3-CRD and prevented by the gal3 inhibitor TD139 in a dose-dependent manner. Additionally, gal3 prevents neuronal network entrainment into proper gamma rhythm. Such disruption is accompanied by the impairment of FSN- and PC-gamma spikephase locking and disturbances of excitatory and inhibitory synaptic transmission. Interestingly, we found a possible functional link for gal3 to AD since 1) TD139 prevents Aß42-induced degradation of gamma oscillations ex-vivo and 2) gamma oscillations are impaired in the CA3 hippocampal area of the 5xFAD mouse model at 6 months-of-age while gamma oscillations recorded from 5xFAD mice lacking gal3 (5xFAD-Gal3KO) remain similar to age-matched WT counterpart. Moreover, our results bridge a gap between cellular and molecular notions on the central role of gal3 in $A D$ progression and behavioral studies by providing missing functional evidence that is relevant for those behaviors. This reinforces the therapeutic potential of inhibiting/removing gal3 to counteract $A D$ progression and putatively for disease-modifying interventions in other neurodegenerative disorders involving microglia activation and neuroinflammation.

\section{List Of Abreviations}

$A_{1} R:$ adenosine receptor 1

ACSF: artificial cerebrospinal fluid

AD: Alzheimer's disease

AP: action potentials

Aß42: beta-amyloid peptide

CNS: Central Nervous System

Cr: coefficient of rhythmicity

CRD: carbohydrate recognition domain

Em: membrane potential

EPSCs: excitatory postsynaptic currents

FSN: fast-spiking interneurons

gal3: galectin 3

IEl: inter-event-interval

IPSCs: inhibitory postsynaptic currents

KA: Kainic acid 
LFP: local field potential

MCl: mild cognitive impairment

PCs: pyramidal cells

TLR4: toll-like receptor 4

TREM2: triggering receptor expressed on myeloid cells 2

WT: wild-type

$\mathrm{XC}$ : cross-correlation index

\section{Declarations}

\section{Ethics approval}

Experiments were performed in accordance with the ethical permit granted by Norra Stockholm's Djurförsöksetiska Nämnd (Dnr N45/13) to AF and Malmö-Lund Ethical Committee on Animal Testing in Sweden (Dnr 5.8.18-01107/2018) to TD.

\section{Consent for publication}

Not applicable

\section{Availability of data and materials}

All the data is provided in the figure legends, in the supplementary material and available upon request without restrictions.

\section{Competing interests}

The authors declare that they have no competing interests.

\section{Funding and Disclosures}

This work was supported by the Swedish Research Council, the Swedish Brain Foundation, the Swedish Alzheimer Foundation, the Åhlén Foundation (AF), the Berger Foundation (TD), the Olle Engkvist Foundation (TD), G\&K Kock Foundation (TD), the Strategic Research Area MultiPark at Lund University (TD), the Foundation for Geriatric Diseases at Karolinska Institutet, the Åhlén Foundation (YAT), Consejo Nacional de Ciencia y Tecnología (CONACYT) postdoctoral fellowships and StratNeuro program at Karolinska Institutet (LEAG), Lindhés Advokabyra AB Grant and Stohnes Stiftelse (LEAG, YAT), the Spanish Ministerio de Ciencia e Innovación (MICIN/AEI/FEDER: PID2019-107677GB-I00; ARM).

Authors' contributions: YAT and LEAG performed electrophysiology and analyzed data. SB performed genotyping and RT-qPCR experiments and analyzed data. AB-S, SB and TD provided the animal models. AB-S 
and TD provided gal3 and gal3 inhibitor. YAT, LEAG and AF designed experiments. AF supervised the study. YAT and AF conceived and wrote the manuscript with input from all authors.

Acknowledgements: We thank Professor Jane Johansson and Dr. Gefei Chen at Karolinska Institutet for providing A 342 peptides.

\section{References}

1. van Vugt MK, Schulze-Bonhage A, Litt B, Brandt A, Kahana MJ. Hippocampal Gamma Oscillations Increase with Memory Load. J Neurosci. 2010;30:2694-9.

2. Buzsáki G. Rythms of the Brain. Oxford University Press; 2011.

3. Womelsdorf T, Fries P. The role of neuronal synchronization in selective attention. Curr. Opin. Neurobiol. 2007. p. 154-60.

4. Howard MW. Gamma Oscillations Correlate with Working Memory Load in Humans. Cereb Cortex [Internet]. 2003;13:1369-74. Available from: http://www.ncbi.nlm.nih.gov/pubmed/14615302.

5. Sederberg PB, Schulze-Bonhage A, Madsen JR, Bromfield EB, McCarthy DC, Brandt A, et al. Hippocampal and neocortical gamma oscillations predict memory formation in humans. Cereb Cortex [Internet]. 2007;17:1190-6. Available from: http://www.ncbi.nlm.nih.gov/pubmed/16831858.

6. Fisahn A, Pike FG, Buhl EH, Paulsen O. Cholinergic induction of network oscillations at $40 \mathrm{~Hz}$ in the hippocampus in vitro. Nature. 1998;394:186-9.

7. Cardin JA, Carlén M, Meletis K, Knoblich U, Zhang F, Deisseroth K, et al. Driving fast-spiking cells induces gamma rhythm and controls sensory responses. Nature [Internet]. 2009;459:663-7. Available from: http://www.nature.com/articles/nature08002.

8. Mann EO, Radcliffe CA, Paulsen O. Hippocampal gamma-frequency oscillations: From interneurones to pyramidal cells, and back. J Physiol. 2005;562:55-63.

9. McBain CJ, Fisahn A. Interneurons unbound. Nat Rev Neurosci [Internet]. 2001;2:11-23. Available from: http://www.ncbi.nlm.nih.gov/pubmed/11253355.

10. Joseph DJ, Liu C, Peng J, Liang G, Wei H. Isoflurane mediated neuropathological and cognitive impairments in the triple transgenic Alzheimer's mouse model are associated with hippocampal synaptic deficits in an age-dependent manner. Ginsberg SD, editor. PLoS One [Internet]. 2019;14:e0223509. Available from: http://www.ncbi.nlm.nih.gov/pubmed/31600350.

11. Csicsvari J, Jamieson B, Wise KD, Buzsáki G. Mechanisms of gamma oscillations in the hippocampus of the behaving rat. Neuron. 2003;37:311-22.

12. Başar E. A review of gamma oscillations in healthy subjects and in cognitive impairment. Int. J. Psychophysiol. 2013. p. 99-117.

13. Stam CJ, van Cappellen van Walsum AM, Pijnenburg YAL, Berendse HW, de Munck JC, Scheltens P, et al. Generalized synchronization of MEG recordings in Alzheimer's Disease: evidence for involvement of the gamma band. J Clin Neurophysiol [Internet]. 2002;19:562-74. Available from: http://www.ncbi.nlm.nih.gov/pubmed/12488788.

14. Guillon J, Attal Y, Colliot O, La Corte V, Dubois B, Schwartz D, et al. Loss of brain inter-frequency hubs in Alzheimer's disease. Sci Rep [Internet]. 2017;7:10879. Available from: 
http://www.ncbi.nlm.nih.gov/pubmed/28883408.

15. Verret L, Mann EO, Hang GB, Barth AMI, Cobos I, Ho K, et al. Inhibitory interneuron deficit links altered network activity and cognitive dysfunction in alzheimer model. Cell [Internet]. NIH Public Access; 2012 [cited 2017 May 16];149:708-21. Available from: http://www.ncbi.nlm.nih.gov/pubmed/22541439.

16. Mucke L, Masliah E, Yu GQ, Mallory M, Rockenstein EM, Tatsuno G, et al. High-level neuronal expression of abeta 1-42 in wild-type human amyloid protein precursor transgenic mice: synaptotoxicity without plaque formation. J Neurosci [Internet]. 2000;20:4050-8. Available from:

http://www.ncbi.nIm.nih.gov/pubmed/10818140.

17. Shankar GM, Li S, Mehta TH, Garcia-Munoz A, Shepardson NE, Smith I, et al. Amyloid- $\beta$ protein dimers isolated directly from Alzheimer's brains impair synaptic plasticity and memory. Nat Med [Internet]. 2008;14:837-42. Available from: http://www.ncbi.nlm.nih.gov/pubmed/18568035.

18. Selkoe DJ. Cell biology of the beta-amyloid precursor protein and the genetics of Alzheimer's disease. Cold Spring Harb Symp Quant Biol [Internet]. 1996;61:587-96. Available from:

http://www.ncbi.nlm.nih.gov/pubmed/9246485.

19. Lasagna-Reeves CA, Castillo-Carranza DL, Sengupta U, Clos AL, Jackson GR, Kayed R. Tau oligomers impair memory and induce synaptic and mitochondrial dysfunction in wild-type mice. Mol Neurodegener [Internet]. 2011;6:39. Available from: http://www.ncbi.nlm.nih.gov/pubmed/21645391.

20. Hoover BR, Reed MN, Su J, Penrod RD, Kotilinek LA, Grant MK, et al. Tau Mislocalization to Dendritic Spines Mediates Synaptic Dysfunction Independently of Neurodegeneration. Neuron [Internet]. 2010;68:1067-81. Available from: http://www.ncbi.nlm.nih.gov/pubmed/21172610.

21. Frere S, Slutsky I. Alzheimer's Disease: From Firing Instability to Homeostasis Network Collapse. Neuron [Internet]. 2018;97:32-58. Available from: http://www.ncbi.nlm.nih.gov/pubmed/29301104.

22. Driscoll I, Resnick SM, Troncoso JC, An Y, O’Brien R, Zonderman AB. Impact of Alzheimer's pathology on cognitive trajectories in nondemented elderly. Ann Neurol [Internet]. 2006;60:688-95. Available from: http://www.ncbi.nlm.nih.gov/pubmed/17192929.

23. Karran E, De Strooper B. The amyloid cascade hypothesis: are we poised for success or failure? J Neurochem [Internet]. 2016;139:237-52. Available from: http://www.ncbi.nlm.nih.gov/pubmed/27255958.

24. Arroyo-García LE, Isla AG, Andrade-Talavera Y, Balleza-Tapia H, Loera-Valencia R, Alvarez-Jimenez L, et al. Impaired spike-gamma coupling of area CA3 fast-spiking interneurons as the earliest functional impairment in the AppNL-G-F mouse model of Alzheimer's disease. Mol Psychiatry [Internet]. Springer US; 2021;1-11. Available from: http://dx.doi.org/10.1038/s41380-021-01257-0.

25. Guillot-Sestier M-V, Doty KR, Gate D, Rodriguez J, Leung BP, Rezai-Zadeh K, et al. II10 Deficiency Rebalances Innate Immunity to Mitigate Alzheimer-Like Pathology. Neuron [Internet]. 2015;85:534-48. Available from: http://www.ncbi.nlm.nih.gov/pubmed/25619654.

26. Boza-Serrano A, Ruiz R, Sanchez-Varo R, García-Revilla J, Yang Y, Jimenez-Ferrer I, et al. Galectin-3, a novel endogenous TREM2 ligand, detrimentally regulates inflammatory response in Alzheimer's disease. Acta Neuropathol [Internet]. 2019;138:251-73. Available from: http://www.ncbi.nlm.nih.gov/pubmed/31006066.

27. Heneka MT, Sastre M, Dumitrescu-Ozimek L, Dewachter I, Walter J, Klockgether T, et al. Focal glial activation coincides with increased BACE1 activation and precedes amyloid plaque deposition in

Page $18 / 33$ 
APP[V717I] transgenic mice. J Neuroinflammation [Internet]. 2005;2:22. Available from: http://www.ncbi.nlm.nih.gov/pubmed/16212664.

28. Heneka MT, Kummer MP, Stutz A, Delekate A, Schwartz S, Vieira-Saecker A, et al. NLRP3 is activated in Alzheimer's disease and contributes to pathology in APP/PS1 mice. Nature [Internet]. 2013;493:674-8. Available from: http://www.ncbi.nlm.nih.gov/pubmed/23254930.

29. Nott A, Holtman IR, Coufal NG, Schlachetzki JCM, Yu M, Hu R, et al. Brain cell type-specific enhancerpromoter interactome maps and disease - risk association. Science (80-) [Internet]. 2019;366:1134-9. Available from: http://www.ncbi.nlm.nih.gov/pubmed/31727856.

30. Schilling S, Chausse B, Dikmen HO, Almouhanna F, Hollnagel J-O, Lewen A, et al. TLR2- and TLR3-activated microglia induce different levels of neuronal network dysfunction in a context-dependent manner. Brain Behav Immun [Internet]. Elsevier Inc.; 2021; Available from: https://doi.org/10.1016/j.bbi.2021.05.013.

31. Chausse B, Lewen A, Poschet G, Kann O. Selective inhibition of mitochondrial respiratory complexes controls the transition of microglia into a neurotoxic phenotype in situ. Brain Behav Immun [Internet]. 2020;88:802-14. Available from: http://www.ncbi.nlm.nih.gov/pubmed/32446944.

32. Burguillos MA, Svensson M, Schulte T, Boza-Serrano A, Garcia-Quintanilla A, Kavanagh E, et al. MicrogliaSecreted Galectin-3 Acts as a Toll-like Receptor 4 Ligand and Contributes to Microglial Activation. Cell Rep [Internet]. 2015;10:1626-38. Available from: http://www.ncbi.nlm.nih.gov/pubmed/25753426.

33. Boza-Serrano A, Reyes JF, Rey NL, Leffler H, Bousset L, Nilsson U, et al. The role of Galectin-3 in asynuclein-induced microglial activation. Acta Neuropathol Commun [Internet]. 2014;2:156. Available from: http://www.ncbi.nlm.nih.gov/pubmed/25387690.

34. De Strooper B, Karran E. The Cellular Phase of Alzheimer's Disease. Cell [Internet]. Elsevier Inc.; 2016;164:603-15. Available from: http://dx.doi.org/10.1016/j.cell.2015.12.056.

35. Dani M, Wood M, Mizoguchi R, Fan Z, Walker Z, Morgan R, et al. Microglial activation correlates in vivo with both tau and amyloid in Alzheimer's disease. Brain [Internet]. 2018;141:2740-54. Available from: http://www.ncbi.nlm.nih.gov/pubmed/30052812.

36. Perlmutter LS, Barron E, Chui HC. Morphologic association between microglia and senile plaque amyloid in Alzheimer's disease. Neurosci Lett [Internet]. 1990;119:32-6. Available from: http://www.ncbi.nlm.nih.gov/pubmed/2097581.

37. Stalder M, Phinney A, Probst A, Sommer B, Staufenbiel M, Jucker M. Association of Microglia with Amyloid Plaques in Brains of APP23 Transgenic Mice. Am J Pathol [Internet]. 1999;154:1673-84. Available from: http://www.ncbi.nlm.nih.gov/pubmed/10362792.

38. Sheffield LG, Marquis JG, Berman NE. Regional distribution of cortical microglia parallels that of neurofibrillary tangles in Alzheimer's disease. Neurosci Lett [Internet]. 2000;285:165-8. Available from: http://www.ncbi.nlm.nih.gov/pubmed/10806312.

39. Serrano-Pozo A, Mielke ML, Gómez-Isla T, Betensky RA, Growdon JH, Frosch MP, et al. Reactive Glia not only Associates with Plaques but also Parallels Tangles in Alzheimer's Disease. Am J Pathol [Internet]. 2011;179:1373-84. Available from: http://www.ncbi.nlm.nih.gov/pubmed/21777559.

40. Heneka MT, Carson MJ, Khoury J, El, Landreth GE, Brosseron F, Feinstein DL, et al. Neuroinflammation in Alzheimer's disease. Lancet Neurol [Internet]. 2015;14:388-405. Available from: http://www.ncbi.nlm.nih.gov/pubmed/25792098. 
41. Pasqualetti G, Brooks DJ, Edison P. The Role of Neuroinflammation in Dementias. Curr Neurol Neurosci Rep [Internet]. 2015;15:17. Available from: http://www.ncbi.nlm.nih.gov/pubmed/25716012.

42. Butovsky O, Weiner HL. Microglial signatures and their role in health and disease. Nat Rev Neurosci [Internet]. 2018;19:622-35. Available from: http://www.ncbi.nlm.nih.gov/pubmed/30206328.

43. Balleza-Tapia H, Crux S, Andrade-Talavera Y, Dolz-Gaiton P, Papadia D, Chen G, et al. TrpV1 receptor activation rescues neuronal function and network gamma oscillations from $A \beta$-induced impairment in mouse hippocampus in vitro. Elife. 2018;7:1-24.

44. Andrade-Talavera Y, Arroyo-García LE, Chen G, Johansson J, Fisahn A. Modulation of Kv3.1/Kv3.2 promotes gamma oscillations by rescuing $A \beta$-induced desynchronization of fast-spiking interneuron firing in an AD mouse model in vitro. J Physiol [Internet]. 2020;JP279718. Available from: https://onlinelibrary.wiley.com/doi/abs/10.1113/JP279718.

45. Kurudenkandy FR, Zilberter M, Biverstal H, Presto J, Honcharenko D, Stromberg R, et al. Amyloid-betaInduced Action Potential Desynchronization and Degradation of Hippocampal Gamma Oscillations Is Prevented by Interference with Peptide Conformation Change and Aggregation. J Neurosci [Internet]. 2014;34:11416-25. Available from: http://www.ncbi.nlm.nih.gov/pubmed/25143621.

46. Gulyas Al, Szabo GG, Ulbert I, Holderith N, Monyer H, Erdelyi F, et al. Parvalbumin-Containing Fast-Spiking Basket Cells Generate the Field Potential Oscillations Induced by Cholinergic Receptor Activation in the Hippocampus. J Neurosci [Internet]. 2010;30:15134-45. Available from: http://www.jneurosci.org/cgi/doi/10.1523/JNEUROSCI.4104-10.2010.

47. Andrade-Talavera Y, Chen G, Kurudenkandy FR, Johansson J, Fisahn A. Bri2 BRICHOS chaperone rescues impaired fast-spiking interneuron behavior and neuronal network dynamics in an AD mouse model in vitro. Neurobiol Dis [Internet]. 2021;159:105514. Available from: https://linkinghub.elsevier.com/retrieve/pii/S0969996121002631.

48. Andrade-Talavera Y, Balleza-Tapia H, Dolz-Gaitón P, Chen G, Johansson J, Fisahn A. Ablation of p75NTR signaling strengthens gamma-theta rhythm interaction and counteracts $A \beta$-induced degradation of neuronal dynamics in mouse hippocampus in vitro. Transl Psychiatry [Internet]. 2021;11:212. Available from: http://www.ncbi.nlm.nih.gov/pubmed/33837176.

49. Hentschke H, Perkins MG, Pearce RA, Banks MI. Muscarinic blockade weakens interaction of gamma with theta rhythms in mouse hippocampus. Eur J Neurosci. 2007;26:1642-56.

50. Lepur A, Salomonsson E, Nilsson UJ, Leffler H. Ligand Induced Galectin-3 Protein Self-association. J Biol Chem [Internet]. 2012;287:21751-6. Available from: http://www.ncbi.nlm.nih.gov/pubmed/22549776.

51. Salomonsson E, Carlsson MC, Osla V, Hendus-Altenburger R, Kahl-Knutson B, Öberg CT, et al. Mutational Tuning of Galectin-3 Specificity and Biological Function. J Biol Chem [Internet]. 2010;285:35079-91. Available from: http://www.ncbi.nlm.nih.gov/pubmed/20807768.

52. Buzsáki G, Draguhn A. Neuronal oscillations in cortical networks. Science. 2004;304:1926-9.

53. Pelkey KA, Chittajallu R, Craig MT, Tricoire L, Wester JC, McBain CJ. Hippocampal GABAergic Inhibitory Interneurons. Physiol Rev. 2017;97:1619-747.

54. Andersson R, Johnston A, Fisahn A. Dopamine D4 receptor activation increases hippocampal Gamma oscillations by enhancing synchronization of fast-spiking interneurons. PLoS One. 2012;7. 
55. Chen G, Abelein A, Nilsson HE, Leppert A, Andrade-Talavera Y, Tambaro S, et al. Bri2 BRICHOS client specificity and chaperone activity are governed by assembly state. Nat Commun. 2017;8.

56. Chen G, Andrade-Talavera Y, Tambaro S, Leppert A, Nilsson HEHE, Zhong X, et al. Augmentation of Bri2 molecular chaperone activity against amyloid- $\beta$ reduces neurotoxicity in mouse hippocampus in vitro. Commun Biol [Internet]. 2020;3:32. Available from: http://www.ncbi.nlm.nih.gov/pubmed/31959875.

57. Goutagny R, Krantic S. Hippocampal Oscillatory Activity in Alzheimer's Disease: Toward the Identification of Early Biomarkers? Number [Internet]. 2013;4:134-40. Available from:

http://www.ncbi.nIm.nih.gov/pubmed/23730529.

58. laccarino HF, Singer AC, Martorell AJ, Rudenko A, Gao F, Gillingham TZ, et al. Gamma frequency entrainment attenuates amyloid load and modifies microglia. Nature [Internet]. 2016;540:230-5. Available from:

http://www.ncbi.nlm.nih.gov/pubmed/27929004\%0Ahttp://www.pubmedcentral.nih.gov/articlerender.fcgi? artid=PMC5656389.

59. Mondragón-Rodríguez S, Gu N, Manseau F, Williams S. Alzheimer's Transgenic Model Is Characterized by Very Early Brain Network Alterations and $\beta$-CTF Fragment Accumulation: Reversal by $\beta$-Secretase Inhibition. Front Cell Neurosci. 2018;12:1-17.

60. Etter G, van der Veldt S, Manseau F, Zarrinkoub I, Trillaud-Doppia E, Williams S. Optogenetic gamma stimulation rescues memory impairments in an Alzheimer's disease mouse model. Nat Commun [Internet]. 2019;10:5322. Available from: http://www.ncbi.nlm.nih.gov/pubmed/31757962.

61. Isla AG, Balleza-Tapia H, Fisahn A. Efficacy of preclinical pharmacological interventions against alterations of neuronal network oscillations in Alzheimer's disease: A systematic review. Exp Neurol [Internet]. Experimental Neurology; 2021;113743. Available from: http://www.ncbi.nlm.nih.gov/pubmed/34000250.

62. Sasaguri H, Nilsson P, Hashimoto S, Nagata K, Saito T, De Strooper B, et al. APP mouse models for Alzheimer's disease preclinical studies. EMBO J [Internet]. 2017;36:2473-87. Available from: http://www.ncbi.nlm.nih.gov/pubmed/28768718.

63. Crouzin N, Baranger K, Cavalier M, Marchalant Y, Cohen-Solal C, Roman FS, et al. Area-Specific Alterations of Synaptic Plasticity in the 5XFAD Mouse Model of Alzheimer's Disease: Dissociation between Somatosensory Cortex and Hippocampus. Lakshmana MK, editor. PLoS One [Internet]. 2013;8:e74667. Available from: http://www.ncbi.nlm.nih.gov/pubmed/24069328.

64. Sohal VS, Zhang F, Yizhar O, Deisseroth K. Parvalbumin neurons and gamma rhythms enhance cortical circuit performance. Nature [Internet]. 2009;459:698-702. Available from: http://www.ncbi.nlm.nih.gov/pubmed/19396159.

65. Buzsáki G. Neural Syntax: Cell Assemblies, Synapsembles, and Readers. Neuron [Internet]. 2010;68:36285. Available from: https://linkinghub.elsevier.com/retrieve/pii/S0896627310007658.

66. Poska H, Haslbeck M, Kurudenkandy FR, Hermansson E, Chen G, Kostallas G, et al. Dementia-related Bri2 BRICHOS is a versatile molecular chaperone that efficiently inhibits A 42 toxicity in Drosophila. Biochem J [Internet]. 2016;473:3683-704. Available from: http://www.ncbi.nlm.nih.gov/pubmed/27514716.

67. Park K, Lee J, Jang HJ, Richards BA, Kohl MM, Kwag J. Optogenetic activation of parvalbumin and somatostatin interneurons selectively restores theta-nested gamma oscillations and oscillation-induced 
spike timing-dependent long-term potentiation impaired by amyloid $\beta$ oligomers. BMC Biol [Internet]. 2020;18:7. Available from: https://bmcbiol.biomedcentral.com/articles/10.1186/s12915-019-0732-7.

68. Barondes SH, Castronovo V, Cooper DNW, Cummings RD, Drickamer K, Felzi T, et al. Galectins: A family of animal $\beta$-galactoside-binding lectins. Cell [Internet]. 1994;76:597-8. Available from:

http://www.ncbi.nlm.nih.gov/pubmed/8124704.

69. Leffler H, Carlsson S, Hedlund M, Qian Y, Poirier F. Introduction to galectins. Glycoconj J [Internet]. 2002;19:433-40. Available from: http://www.ncbi.nlm.nih.gov/pubmed/14758066.

70. Stegmayr J, Zetterberg F, Carlsson MC, Huang X, Sharma G, Kahl-Knutson B, et al. Extracellular and intracellular small-molecule galectin-3 inhibitors. Sci Rep [Internet]. 2019;9:2186. Available from: http://www.ncbi.nlm.nih.gov/pubmed/30778105.

71. Agostinho P, Madeira D, Dias L, Simões AP, Cunha RA, Canas PM. Purinergic signaling orchestrating neuron-glia communication. Pharmacol Res [Internet]. 2020;162:105253. Available from: http://www.ncbi.nlm.nih.gov/pubmed/33080321.

72. George J, Cunha RA, Mulle C, Amédée T. Microglia-derived purines modulate mossy fibre synaptic transmission and plasticity through P2X4 and A1 receptors. Eur J Neurosci. 2016;43:1366-78.

73. Rebola N, Simões AP, Canas PM, Tomé AR, Andrade GM, Barry CE, et al. Adenosine A2A receptors control neuroinflammation and consequent hippocampal neuronal dysfunction. J Neurochem [Internet]. 2011;117:100-11. Available from: http://doi.wiley.com/10.1111/j.1471-4159.2011.07178.x.

74. Viana da Silva S, Haberl MG, Zhang P, Bethge P, Lemos C, Gonçalves N, et al. Early synaptic deficits in the APP/PS1 mouse model of Alzheimer's disease involve neuronal adenosine A2A receptors. Nat Commun [Internet]. 2016;7:11915. Available from: http://www.ncbi.nlm.nih.gov/pubmed/27312972.

75. Albasanz JL, Perez S, Barrachina M, Ferrer I, Martín M. Up-regulation of Adenosine Receptors in the Frontal Cortex in Alzheimer's Disease. Brain Pathol [Internet]. 2008;18:211-9. Available from: http://www.ncbi.nlm.nih.gov/pubmed/18241242.

76. Orr AG, Hsiao EC, Wang MM, Ho K, Kim DH, Wang X, et al. Astrocytic adenosine receptor A2A and Gscoupled signaling regulate memory. Nat Neurosci [Internet]. 2015;18:423-34. Available from: http://www.ncbi.nlm.nih.gov/pubmed/25622143.

77. Pietersen AN, Lancaster DM, Patel N, Hamilton JB, Vreugdenhil M. Modulation of gamma oscillations by endogenous adenosine through $\mathrm{A} 1$ and $\mathrm{A} 2 \mathrm{~A}$ receptors in the mouse hippocampus. Neuropharmacology [Internet]. Elsevier Ltd; 2009;56:481-92. Available from: http://dx.doi.org/10.1016/j.neuropharm.2008.10.001.

78. Schulz SB, Klaft Z-J, Rösler AR, Heinemann U, Gerevich Z. Purinergic P2X, P2Y and adenosine receptors differentially modulate hippocampal gamma oscillations. Neuropharmacology [Internet]. Elsevier Ltd; 2012;62:914-24. Available from: http://dx.doi.org/10.1016/j.neuropharm.2011.09.024.

79. Li H, Henry JL. Adenosine action on interneurons and synaptic transmission onto interneurons in rat hippocampus in vitro. Eur J Pharmacol [Internet]. 2000;407:237-44. Available from: http://www.ncbi.nlm.nih.gov/pubmed/11068019.

80. Schubert P, Heinemann U, Kolb R. Differential effect of adenosine on pre- and postsynaptic calcium fluxes. Brain Res [Internet]. 1986;376:382-6. Available from: https://linkinghub.elsevier.com/retrieve/pii/0006899386902040. 
81. Ulland TK, Song WM, Huang SC-C, Ulrich JD, Sergushichev A, Beatty WL, et al. TREM2 Maintains Microglial Metabolic Fitness in Alzheimer's Disease. Cell [Internet]. 2017;170:649-663.e13. Available from: http://www.ncbi.nlm.nih.gov/pubmed/28802038.

82. Goutagny R, Gu N, Cavanagh C, Jackson J, Chabot JG, Quirion R, et al. Alterations in hippocampal network oscillations and theta-gamma coupling arise before $A \beta$ overproduction in a mouse model of Alzheimer's disease. Eur J Neurosci. 2013;37:1896-902.

83. Selkoe DJ. Alzheimer's disease is a synaptic failure. Science [Internet]. 2002;298:789-91. Available from: http://www.ncbi.nlm.nih.gov/pubmed/12399581.

84. Andrade-Talavera Y, Rodríguez-Moreno A. Synaptic Plasticity and Oscillations in Alzheimer's Disease: A Complex Picture of a Multifaceted Disease. Front Mol Neurosci [Internet]. 2021;14:696476. Available from: https://www.frontiersin.org/articles/10.3389/fnmol.2021.696476/full.

85. Boza-Serrano A, Yang Y, Paulus A, Deierborg T. Innate immune alterations are elicited in microglial cells before plaque deposition in the Alzheimer's disease mouse model 5xFAD. Sci Rep [Internet]. 2018;8:1550. Available from: http://www.nature.com/articles/s41598-018-19699-y.

86. Venegas C, Kumar S, Franklin BS, Dierkes T, Brinkschulte R, Tejera D, et al. Microglia-derived ASC specks cross-seed amyloid- $\beta$ in Alzheimer's disease. Nature [Internet]. 2017;552:355-61. Available from: http://www.ncbi.nlm.nih.gov/pubmed/29293211.

87. Sosna J, Philipp S, Albay R, Reyes-Ruiz JM, Baglietto-Vargas D, LaFerla FM, et al. Early long-term administration of the CSF1R inhibitor PLX3397 ablates microglia and reduces accumulation of intraneuronal amyloid, neuritic plaque deposition and pre-fibrillar oligomers in 5XFAD mouse model of Alzheimer's disease. Mol Neurodegener [Internet]. 2018;13:11. Available from: http://www.ncbi.nlm.nih.gov/pubmed/29490706.

88. Ta T-T, Dikmen HO, Schilling S, Chausse B, Lewen A, Hollnagel J-O, et al. Priming of microglia with IFN-Y slows neuronal gamma oscillations in situ. Proc Natl Acad Sci [Internet]. 2019;116:4637-42. Available from: http://www.pnas.org/lookup/doi/10.1073/pnas.1813562116.

\section{Figures}


Figure 1
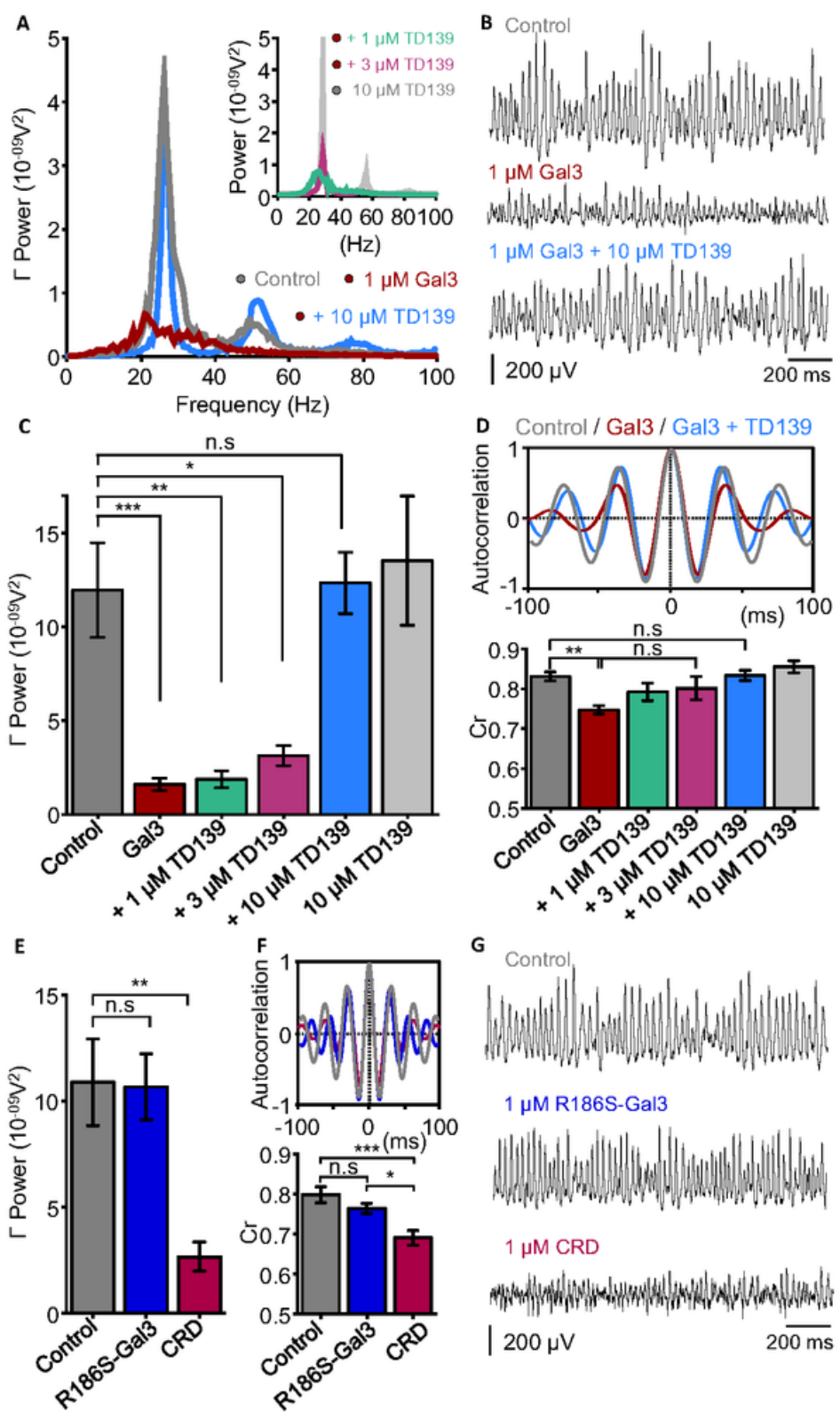

G
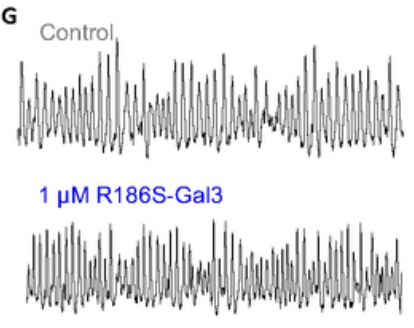

$1 \mu \mathrm{M}$ CRD

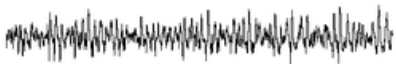

| $200 \mu \mathrm{V} \quad 20 \overline{\mathrm{ms}}$

\section{Figure 1}

Gal3 impairs gamma oscillation power and rhythmicity through its carbohydrate recognition-domain (CRD).

A) Representative power spectra of hippocampal CA3 network activity recorded in control slices (gray), slices pre-incubated for 15 min with $1 \mu \mathrm{M}$ Gal3 (red) and slices co-incubated with $1 \mu \mathrm{M} \mathrm{Gal3} \mathrm{+} 10 \mu \mathrm{M}$ TD139 (blue). Inset: Representative power spectra for slices co-incubated with $1 \mu \mathrm{M} \mathrm{Gal3}+10 \mu \mathrm{M}$ TD139 (gray), co-incubated with $1 \mu \mathrm{M} \mathrm{Gal3}+3 \mu \mathrm{M}$ TD139 (magenta) or co-incubated with $1 \mu \mathrm{M} \mathrm{Gal3}+1 \mu \mathrm{M}$ TD139 (green). B) Representative example traces of recordings performed in the conditions shown in A. C) Summary bar graphs 
of gamma oscillation power for the conditions shown in A demonstrating that $10 \mu \mathrm{M}$ TD139 confers the most effective prevention against gal3-induced decrease of gamma oscillation power (ordinary one-way ANOVA followed by Holm-Sidak's multiple comparisons test, Table S1): control (gray, $12.0 \pm 2.52 \times 10^{-09} \mathrm{~V}^{2}, \mathrm{n}=14$ ); gal3 (red, $1.60 \pm 0.33 \times 10^{-09} \mathrm{~V}^{2}, \mathrm{n}=13$ ); gal3 $+1 \mu \mathrm{M}$ TD139 (green, $1.88 \pm 0.45 \times 10^{-09} \mathrm{~V}^{2}, \mathrm{n}=10$ ); gal3 $+3 \mu \mathrm{M}$ TD139 (magenta, $3.13 \pm 0.53 \times 10^{-09} \mathrm{~V}^{2}, \mathrm{n}=8$ ); gal3 + $10 \mu \mathrm{M}$ TD139 (blue, $12.3 \pm 1.63 \times 10^{-09} \mathrm{~V}^{2}, \mathrm{n}=14$ ). $10 \mu \mathrm{M}$ TD139 applied alone does not affect gamma oscillations power (light gray, $13.5 \pm 3.44 \times 10^{-09} \mathrm{~V}^{2}, \mathrm{n}=6$ ). D) Coefficient of rhythmicity calculated from the autocorrelation function as a measure of gamma oscillation quality (see methods). Top: Representative autocorrelation of gamma oscillations recorded in control conditions (gray), in slices pre-incubated with gal3 (red) and slices co-incubated with gal3 + $10 \mu \mathrm{M}$ TD139 (blue). Bottom: Bar graphs summarizing the $\mathrm{Cr}$ calculated for each condition listed in $\mathrm{C}$ : control (gray, $0.83 \pm$ 0.01, $\mathrm{n}=14$ ); gal3 (red, $0.75 \pm 0.01, \mathrm{n}=13$ ); gal3 + $1 \mu \mathrm{M}$ TD139 (green, $0.79 \pm 0.02, \mathrm{n}=10$ ); gal3 + $3 \mu \mathrm{M}$ TD139 (magenta, $0.8 \pm 0.03, n=8$ ); gal3 $+10 \mu \mathrm{M}$ TD139 (blue, $0.83 \pm 0.01, n=14$ ). $10 \mu \mathrm{M}$ TD139 applied alone does not affect gamma oscillation rhythmicity (light gray, $0.85 \pm 0.02, n=6$ ). Statistical testing performed by ordinary one-way ANOVA followed by Holm-Sidak's multiple comparisons test (Table S2). E) Bar graph summary of gamma oscillation power from slices recorded in control conditions (gray, $10.9 \pm 2.04 \times 10^{-09} \mathrm{~V}^{2}, \mathrm{n}=12$ ), slices pre-incubated 15 min with $1 \mu \mathrm{M} \mathrm{R} 186 \mathrm{~S}$-Gal3 (blue, $10.7 \pm 1.56 \times 10^{-09} \mathrm{~V}^{2}, \mathrm{n}=10 ; \mathrm{p}=0.9265 \mathrm{vs}$. control) and slices pre-incubated 15 min with $1 \mu \mathrm{M}$ CRD-Gal3 (red, $2.67 \pm 0.69 \times 10^{-09} \mathrm{~V}^{2}, n=10 ; p=0.0022$ vs. control, $p=$ 0.0029 vs. R186S-Gal3), ordinary one-way ANOVA followed by Holm-Sidak's multiple comparisons test. F) Top: Representative autocorrelations of gamma oscillations recorded in the conditions mentioned in E. Bottom: Summary bar graphs of the $\mathrm{Cr}$ measured in control conditions (gray, $0.8 \pm 0.02, n=12$ ); slices pre-incubated with R186S-Gal3 (blue, $0.76 \pm 0.01, n=10 ; p=0.1866$ vs. control), and slices pre-incubated with Gal3-CRD (red, $0.69 \pm 0.02, n=11 ; p=0.0004$ vs. control, $p=0.0159$ vs. R186S-Gal3), ordinary one-way ANOVA followed by Holm-Sidak's multiple comparisons test. G) Representative example traces of recordings performed in the conditions shown in E and F. Data is presented as mean \pm SE. Significance levels are shown as * $p<0.05$, ** $p<0.01, * \star \star p<0.001$. 
Figure 2
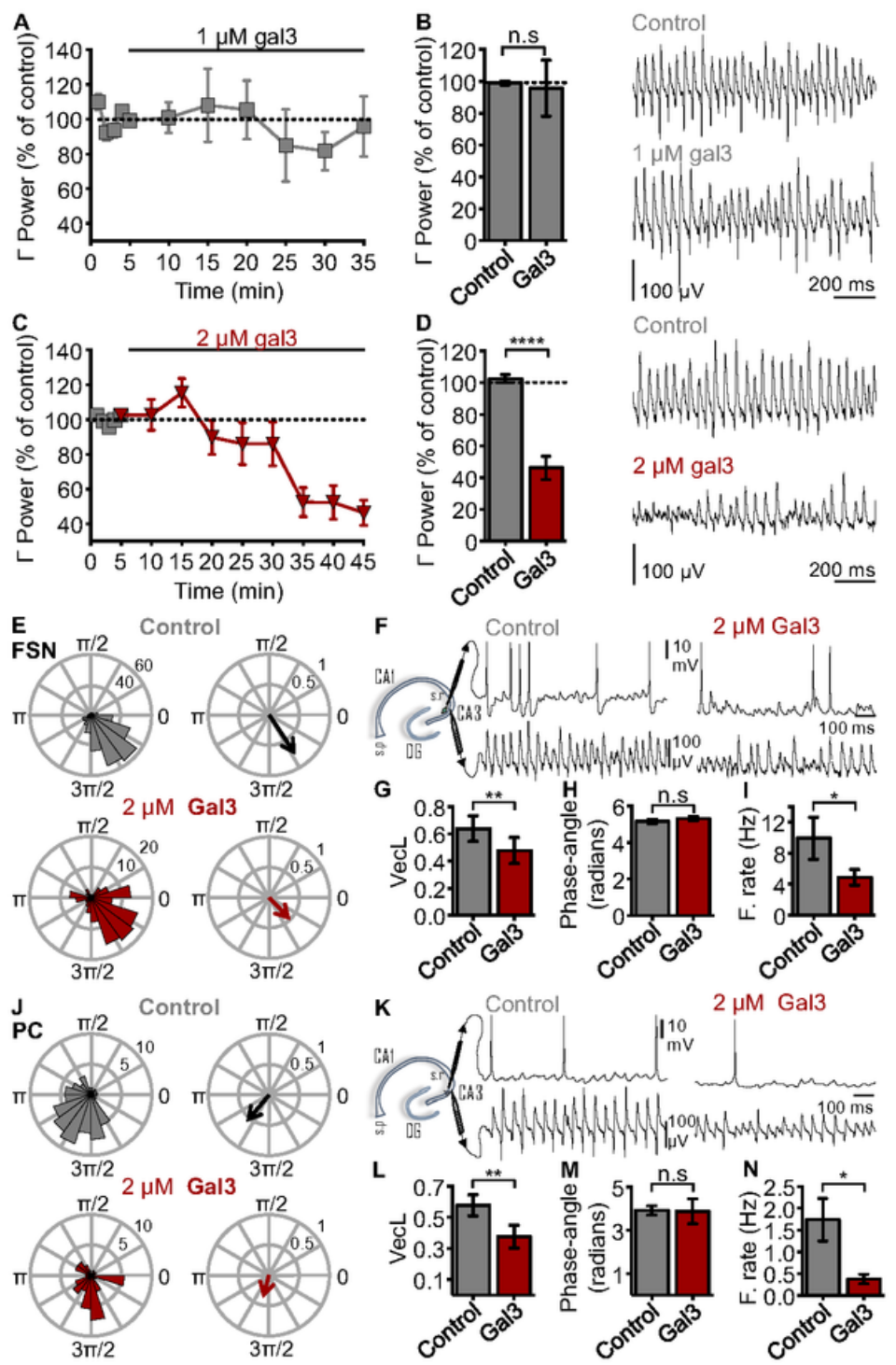

Figure 2

\section{Gal3-induced impairment of FSN and PC action potential phase lock to gamma oscillations.}

A) Time course of the effect of $1 \mu \mathrm{M}$ gal3 application on gamma oscillation power. B) Left: Summary of gamma power in control condition (99.2 $\pm 1.25 \%$ ) and 30 min after $1 \mu \mathrm{M}$ gal3 application $(95.8 \pm 17.5 \%, n=6$; p>0.9999 vs. control), two-tailed Wilcoxon test. Right: Representative example traces of the conditions shown in the bar graph. C) Time course of the effect of $2 \mu \mathrm{M}$ gal3 application on gamma oscillation power. D) Left: Summary of gamma power in control condition (gray, $102.6 \pm 2.6 \%$ ) and 40 min after $2 \mu \mathrm{M}$ gal3 application 
(46.4 $\pm 7.39 \%, n=14 ; p>0.9999)$, two-tailed Wilcoxon test. Right: Representative example traces of the conditions shown in the bar graph. E) Representative polar plots of the AP firing window for a FSN recorded concomitantly to gamma oscillations in control conditions (gray) and 40 min after $2 \mu \mathrm{M}$ gal3 application (red). Left: FSN-AP firing window relative to gamma. Right: resultant vector showing the magnitude of the phase-lock and the gamma phase-angle preference. F) Representative example traces of FSN AP firing (upper traces) concomitantly recorded with gamma oscillations (lower traces) in conditions mentioned in E and D. Left: schematic of the hippocampus showing the locations of FSN AP and LFP recordings. G) Quantification of the vector length (control: $0.64 \pm 0.1$, gal3: $0.48 \pm 0.1, n=9 ; p=0.0092$ ), two-tailed t-test. $H$ ) Quantification of the gamma-preferred phase-angle (control: $5.2 \pm 0.13$ radians, gal3: $5.3 \pm 0.13$ radians, $n=9 ; p=0.1459$ ), two-tailed t-test. I) Quantification of the FSN firing rate (control: $9.91 \pm 2.73 \mathrm{~Hz}$, gal3: $4.9 \pm 1, n=9 ; p=0.0389$ ), two-tailed ttest. J) Representative polar plots of the AP firing window for a PC recorded concomitantly to gamma oscillations in control conditions (gray) and 40 min after $2 \mu \mathrm{M}$ gal3 application (red). Left: PC AP firing window relative to gamma. Right: resultant vector showing the magnitude of the phase-lock and the gamma phaseangle preference. $\mathrm{K}$ ) Representative example traces of PC AP firing (upper traces) concomitantly recorded with gamma oscillations (lower traces) in conditions mentioned in $J$ and K. L) Quantification of the vector length (control: $0.58 \pm 0.1$, gal3: $0.38 \pm 0.1, n=7 ; p=0.0078$ ), one-tailed Wilcoxon test. M) Quantification of the gamma-preferred phase-angle (control: $3.9 \pm 0.21$, gal3: $3.9 \pm 0.58, n=7 ; p=0.5781$ ), two-tailed Wilcoxon test. N) Quantification of the PC firing rate (control: $1.74 \pm 0.5 \mathrm{~Hz}$, gal3: $0.38 \pm 0.11, n=11 ; p=0.0389$ ), paired two-

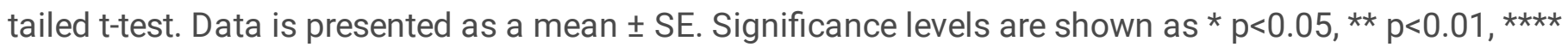
$p<0.0001$. IEl: Inter-event interval. 

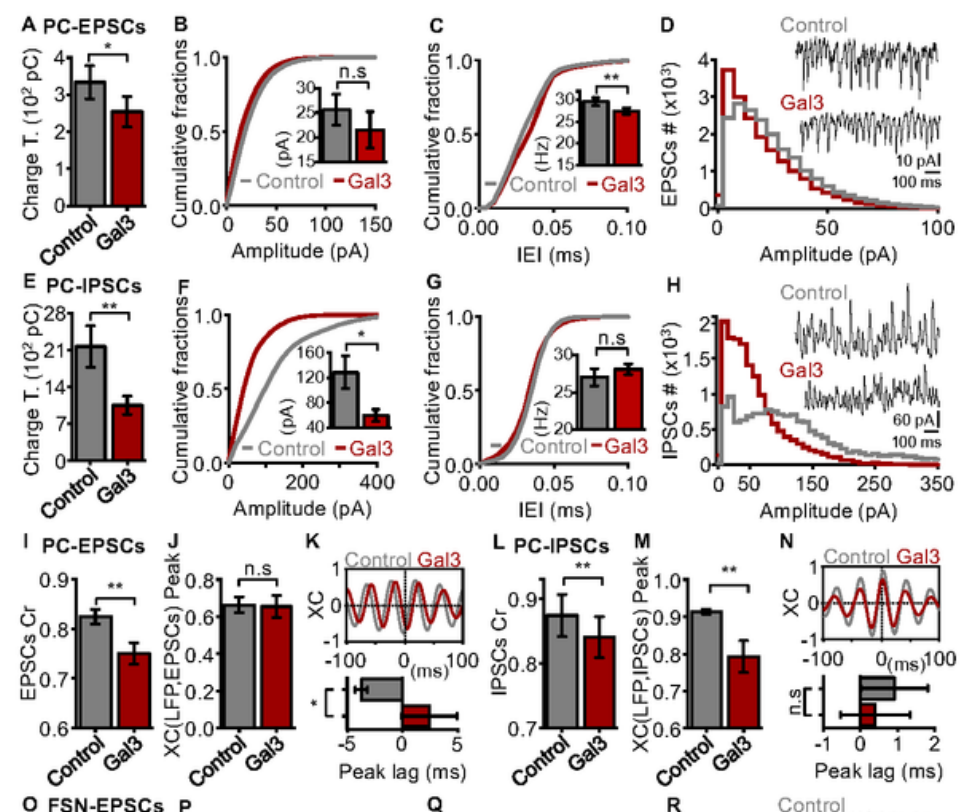

K 1 Control Gal3

L PC-IPSCs M

$N$

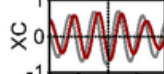

$\begin{array}{ll}-1 & \\ -100 & 0 \\ (\mathrm{~ms}) & 100\end{array}$
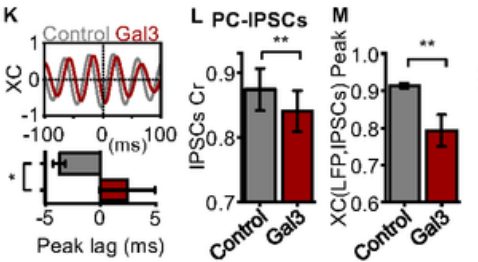

(1)

O FSN-EPSCS

Peak lag (ms)
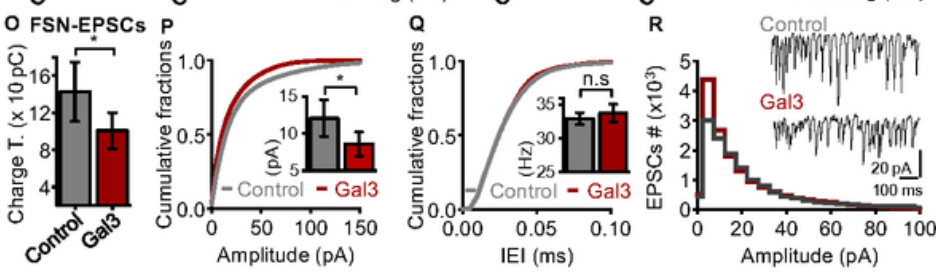

\section{Figure 3}

\section{Gal3-induced impairment of excitatory and inhibitory synaptic transmission during gamma oscillations.}

A) Summary bar graphs of EPSC charge transfer in PCs in control conditions (gray) and 40 min after $2 \mu \mathrm{M}$ gal3 application (red) (control: $333.2 \pm 44.4$ pC, gal3: $253.9 \pm 41.3 p C, n=11 ; p=0.0423$ ), paired one-tailed t-test. B) Cumulative probability of PC EPSC amplitude in control conditions (gray) and 40 min after $2 \mu \mathrm{M}$ gal3 application (red). Inset: Quantification of mean amplitude (control: $25.7 \pm 3.1$ pA, gal3: $21.5 \pm 3.68$ pA, $n=11 ; p=$ 0.2158), paired two-tailed t-test. C) Cumulative probability of PC EPSC frequency in control conditions (gray) 
and 40 min after $2 \mu \mathrm{M}$ gal3 application (red). Inset: Quantification of mean frequency (control: $29.6 \pm 0.91 \mathrm{~Hz}$, gal3: $27.4 \pm 0.79 \mathrm{~Hz}, \mathrm{n}=11 ; \mathrm{p}=0.0046$ ), paired two-tailed t-test. D) EPSC amplitude distribution in conditions mentioned in A. Inset: Representative traces of EPSC recordings for each condition. E) Summary bar graphs of IPSC charge transfer in PCs in control conditions (gray) and 40 min after $2 \mu \mathrm{M}$ gal3 application (red) (control: $2170 \pm 395.4$ pC, gal3: $1052 \pm 171.3$ pC, $n=9 ; p=0.0049$ ), paired one-tailed t-test. F) Cumulative probability of PC IPSC amplitude in control conditions (gray) and 40 min after $2 \mu \mathrm{M}$ gal3 application (red). Inset:

Quantification of mean amplitude (control: $128.8 \pm 26.3$ pA, gal3: $59.5 \pm 9.72$ pA, $n=9 ; p=0.0143$ ), paired twotailed t-test. G) Cumulative probability of PC IPSC frequency in control conditions (gray) and 40 min after $2 \mu \mathrm{M}$ gal3 application (red). Inset: Quantification of mean frequency (control: $27.0 \pm 1.17 \mathrm{~Hz}$, gal3: $28.1 \pm 0.74 \mathrm{~Hz}$, n= 9; $p=0.2004)$, paired two-tailed t-test. H) IPSCs amplitude distribution in conditions mentioned in E. Inset: Representative traces of IPSC recordings for each condition. I) Coefficient of rhythmicity calculated from PC EPSCs in the conditions described in A (control: $0.82 \pm 0.02$, gal3: $0.75 \pm 0.02, n=11 ; p=0.0040$ ), paired twotailed t-test. J) Analysis of the cross-correlation (XC) (LFP, EPSCs) peak size reveals that gal3 does not alter the LFP-EPSC similarity (control: $0.66 \pm 0.04$, gal3: $0.65 \pm 0.06, n=11 ; p=0.8994$ ), two-tailed Wilcoxon test. $K$ ) Top: Representative cross-correlation (XC) calculation performed between gamma LFP and EPSC signals in the conditions described in A. Bottom: Analysis of the peak lag of the XC (LFP, EPSCs) reveals that gal3 induces a phase shift in the maximal coordination between LFP and EPSCs (control: $-3.71 \pm 0.54 \mathrm{~ms}$, gal3: $2.43 \pm 2.53$, n= $7 ; p=0.0156)$, two-tailed Wilcoxon test. L) Coefficient of rhythmicity calculated from PC IPSCs in the conditions described in E showing that gal3 drastically affects IPSC rhythmicity (control: $0.87 \pm 0.03$, gal3: $0.84 \pm 0.03$, n= 7; $p=0.0078$ ), paired one-tailed Wilcoxon test. M) Quantification of the XC (LFP, IPSCs) peak size showing that gal3 impairs LFP-IPSC similarity (control: $0.91 \pm 0.01$, gal3: $0.79 \pm 0.04, n=7 ; p=0.0078$ ), paired one-tailed Wilcoxon test. N) Top: Representative cross-correlation (XC) calculation performed between gamma LFP and IPSC signals. Bottom: Analysis of the peak lag of the XC (LFP, IPSCs) reveals that gal3 induces a nonsignificant phase shift in the maximal coordination between LFP and IPSCs (control: $0.91 \pm 0.91 \mathrm{~ms}, \mathrm{gal} 3: 0.4 \pm$ 0.94, $n=7 ; p=0.2969$ ), two-tailed Wilcoxon test. 0) Quantification of the excitatory charge transfer (control: $142.8 \pm 31.8$ pC, gal3: $100.7 \pm 19.6$ pC, $n=7 ; p=0.0146$ ), paired one-tailed Wilcoxon test. P) Cumulative probability of FSN EPSC amplitude in control conditions (gray) and 40 min after $2 \mu \mathrm{M}$ gal3 application (red). Inset: Quantification of mean amplitude (control: $12.1 \pm 2.5$ pA, gal3: $8.59 \pm 1.63$ pA, n= 7; $p=0.0313$ ), paired two-tailed Wilcoxon test. Q) Cumulative probability of FSN EPSC frequency in control conditions (gray) and 40 min after $2 \mu \mathrm{M}$ gal3 application (red). Inset: Quantification of mean frequency (control: $33.0 \pm 0.89 \mathrm{~Hz}$, gal3: $33.8 \pm 1.35 \mathrm{~Hz}, \mathrm{n}=7$; $\mathrm{p}=0.6875$ ), paired two-tailed Wilcoxon test. R) FSN EPSC amplitude distribution in the conditions mentioned in 0 . Inset: Representative traces of EPSC recordings for each condition. Data is presented as a mean \pm SE. Significance levels are shown as * $p<0.05, * \star p<0.01$. 

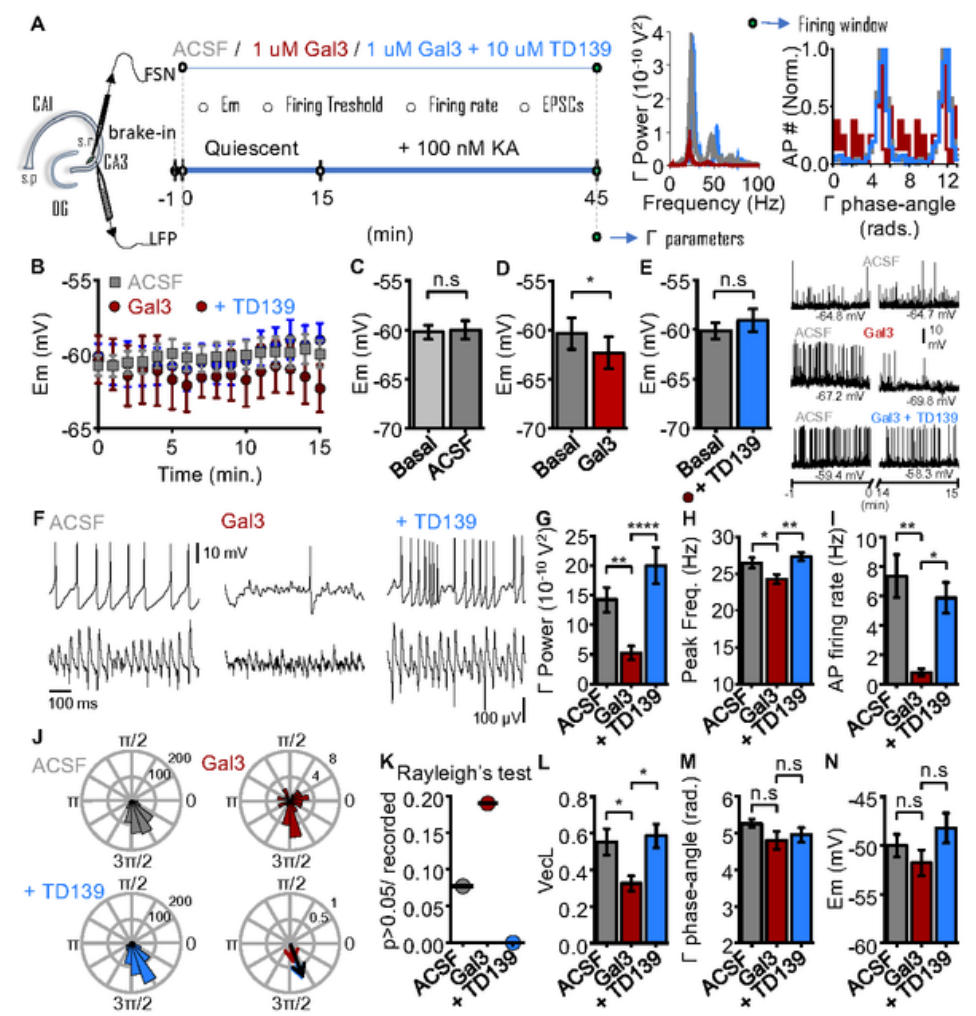

Figure 4

Neuronal mechanisms underlying the activity-dependence of gal3-induced impairment of gamma oscillations.

A) Left: General schematic representation of the experimental set up: B-E correspond to recordings of FSN membrane potential $(\mathrm{Em})$ performed in the quiescent network state for $15 \mathrm{~min}$. Additional measurements for each condition (control, gal3 or gal3 + TD139 application) such as firing at basal Em and firing threshold are provided in Fig. S3. Effects on EPSCs in basal state are provided in Fig. S4. F-N correspond to FSN-gamma phase-lock analyzed from concomitant recordings of the same FSN and gamma network activity for 30 min 
after $100 \mathrm{nM} \mathrm{KA}$ application to induce stable gamma oscillations. Additional measurements for each condition in the activated state such as FSN EPSCs are provided in Fig. S5. Note that both recording electrodes (whole cell FSN patch clamp and LFP recording) are positioned and left in place for the entire experiment (left hippocampal diagram) including gal3 or gal3 + TD139 applications during the quiescent state recordings (first $15 \mathrm{~min}$ ) and during the subsequent gamma induction (following $30 \mathrm{~min}$ ). Right: Representative power spectra of network activity $(0-100 \mathrm{~Hz})$ and representative FSN AP firing windows relative to concomitant gamma oscillation 30 min after KA application for the color-coded experimental conditions shown on the left. B) Time course of the effect of 15 min wash-in of $1 \mu \mathrm{M}$ gal3 (red) or co-application of $1 \mu \mathrm{M}$ gal3 $+10 \mu \mathrm{M}$ TD139 (blue) on FSN membrane potential (Em) in the quiescent network state. Basal control condition (ACSF) is shown in gray. C, D and $\mathbf{E}$ show the quantification of FSN Em over $15 \mathrm{~min}$ of control recordings (basal: $-60.2 \pm 0.73 \mathrm{mV}$, ACSF: $-60.0 \pm 0.92 \mathrm{mV}, \mathrm{n}=18 ; \mathrm{p}=0.7427$ ), after gal3 (basal: $-60.4 \pm 1.61 \mathrm{mV}$, gal3: $-62.3 \pm 1.60 \mathrm{mV}, \mathrm{n}=10 ; \mathrm{p}=$ 0.0346 ) or gal3 + TD139 (basal: $-60.1 \pm 0.82 \mathrm{mV}$, gal3: $-59.0 \pm 1.14 \mathrm{mV}, \mathrm{n}=10 ; \mathrm{p}=0.1804$ ), respectively. Statistics performed: two-tailed paired t-test. Inset: Example traces showing 1 min recorded in basal (control) condition and the last minute recorded for the quantification of the effect of gal3 (red), gal3 + TD139 (blue) as well as the control recorded just with ACSF (gray). Corresponding effects on AP firing for each condition when the FSN was firing at basal Em are shown in Fig. S1. F) Representative traces of concomitant recordings (upper: APs, lower: gamma oscillations) in control conditions as well as in the presence of gal3 or gal3 + TD139. G) Summary of gamma oscillation power calculated from $20-80 \mathrm{~Hz}$ for each condition showing that coapplication of TD139 counteracts the gal3-induced decrease of gamma oscillation power (ACSF: $1.43 \pm 0.21 \mathrm{x}$ $10^{-09} \mathrm{~V}^{2}, \mathrm{n}=15$; gal3: $0.52 \pm 0.12 \times 10^{-09} \mathrm{~V}^{2}, \mathrm{n}=15 ; \mathrm{p}=0.0050$ vs. control, $\mathrm{p}<0.0001$ vs. gal3 + TD139, gal3 + TD 139: $2 \pm 0.31 \times 10^{-09} \mathrm{~V}^{2}, n=11 ; p=0.0644 \mathrm{vs}$. control). H) Summary of gamma peak frequency showing that co-application of TD139 counteracts the gal3-induced slowing of the gamma rhythm (ACSF: $26.5 \pm 0.71 \mathrm{~Hz}, \mathrm{n}=$ 15; gal3: $24.3 \pm 0.63 \mathrm{~Hz}, n=15 ; p=0.0372$ vs. control, $p<0.0099$ vs. gal3 + TD139, gal3 + TD139: $27.3 \pm 0.59$ $\mathrm{Hz}, \mathrm{n}=11 ; \mathrm{p}=0.3867 \mathrm{vs}$. control). I) Summary of AP firing rate showing that gal3-induced AP rate decrease is prevented by co-application of TD139 (ACSF: $7.36 \pm 1.47 \mathrm{~Hz}, \mathrm{n}=12$; gal3: $0.79 \pm 0.26 \mathrm{~Hz}, \mathrm{n}=9$; $\mathrm{p}=0.0012 \mathrm{vs}$. control, $p<0.0118$ vs. gal3 + TD139, gal3 + TD139: $5.88 \pm 1 \mathrm{~Hz}, n=10 ; p=0.3619$ vs. control). J) Representative polar plots of the firing windows shown in A for each condition with the resultant vector (bottom right) showing the magnitude of the FSN-gamma phase-lock and the phase-angle preference for each condition. K) Ratio between the FSN that did not pass the Raileigh's test for uniformity and the total of FSN recorded in the study, showing that in the presence of gal 3 considerably fewer FSN are able to engage in a patterned firing locked to a specific gamma phase (ACSF: 1 out of 15 recorded cells, gal3: 4 out of 14 recorded cells, gal3 + TD139: 0 out of 10 recorded cells). L) Summary of the resultant vector length for each condition (ACSF: $0.55 \pm 0.07, n=12$; gal3: $0.33 \pm 0.04, n=9 ; p=0.0364$ vs. control, $p<0.0308$ vs. gal3 + TD139, gal3 + TD139: $0.59 \pm 0.06, n=10 ; p=$ 0.7143 vs. control). M) Quantification of the phase-angle firing preference revealing that neither gal 3 nor gal $3+$ TD139 induce significant changes (ACSF: $5.26 \pm 0.11$ radians, $n=12$; gal3: $4.81 \pm 0.24$ radians, $n=9 ; p=0.2328$ vs. control, $p=0.5881$ vs. gal3 + TD139, gal3 + TD139: $5 \pm 0.2, n=10 ; p=0.3921$ vs. control). N) Summary bar graphs showing that $30 \mathrm{~min}$ of KA application does not differentially depolarize FSN either in control conditions, or in the presence of gal3 or gal3 + TD139 (ACSF: $-50 \pm 1.17 \mathrm{mV}, \mathrm{n}=12$; gal3: $-51.8 \pm 1.32 \mathrm{mV}, \mathrm{n}=9$; $p=0.5595$ vs. control, $p=0.2304$ vs. gal3 + TD139, gal3 + TD139: $-48.2 \pm 1.54 \mathrm{mV}, n=10 ; p=0.5595$ vs. control). Data is presented as a mean \pm SE. Statistics performed: ordinary one-way ANOVA followed by Holm-Sidak's multiple comparisons test. Significance levels are shown as * $p<0.05$, ${ }^{\star \star} p<0.01,{ }^{\star \star \star \star} p<0.0001$. 
Figure 5
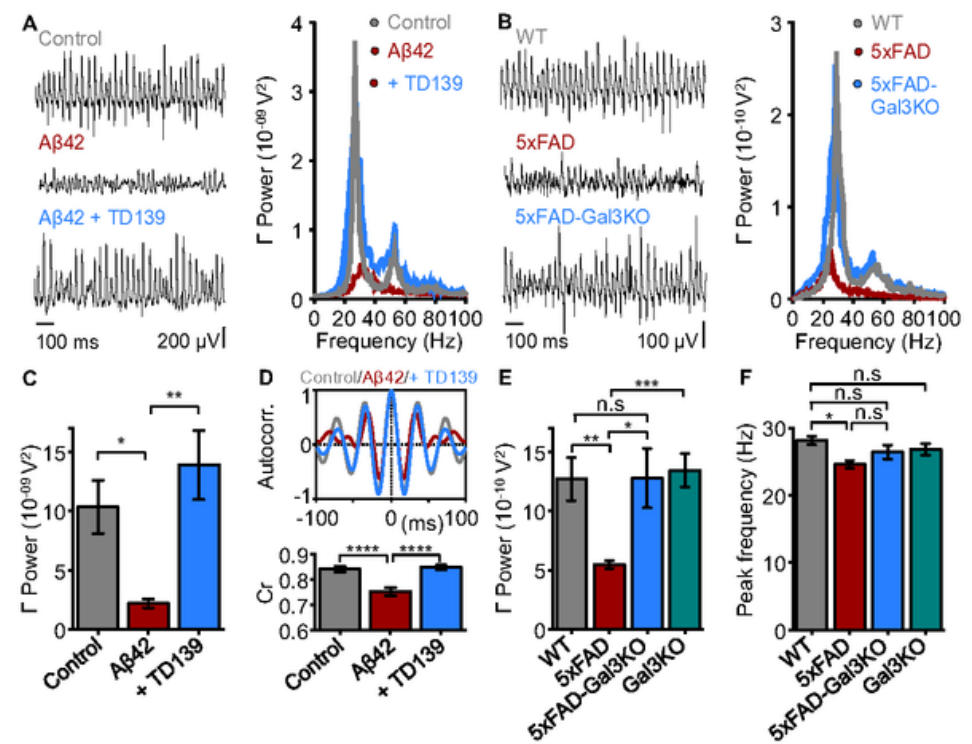

\section{Figure 5}

Involvement of gal3 in the disruption of gamma oscillations in two different AD-related mouse models.

A) Left: Representative example traces of gamma oscillations recorded in an interface-type recording chamber in control conditions (gray) and in slices pre-incubated for 15 min with $50 \mathrm{nM} \mathrm{A \beta 42} \mathrm{(red)} \mathrm{or} 50 \mathrm{nM} \mathrm{A \beta 42+10}$ 4M TD139 (blue). Right: Representative power spectra from network activity $(0-100 \mathrm{~Hz})$ in CA3 hippocampus for each condition. B) Left: Representative example traces of gamma oscillations recorded in CA3 of hippocampal slices from WT (gray), 5xFAD (red) and 5xFAD-Gal3KO (blue) mice in a submerged-type recording 
chamber. Right: Representative power spectra from network activity $(0-100 \mathrm{~Hz})$ in CA3 hippocampus for each animal group. C) Summary of gamma oscillation power $(20-80 \mathrm{~Hz})$ showing that TD139 counteracts A $342-$ induced decrease of gamma power (control: $10.4 \pm 2.24 \times 10^{-09} \mathrm{~V}^{2}, \mathrm{n}=8 ; A \beta 42: 2.23 \pm 0.36 \times 10^{-09} \mathrm{~V}^{2}, \mathrm{n}=8 ; \mathrm{p}=$ 0.0478 vs. control, $p=0.0040$ vs. $A \beta 42+T D 139, A \beta 42+T D 139: 13.9 \pm 2.9 \times 10^{-09} V^{2}, n=10 ; p=0.2809$ vs. control). D) Summary of the $\mathrm{Cr}$ showing that TD139 prevents $A \beta 42$-induced deterioration of gamma rhythmicity (control: $0.84 \pm 0.01, n=8 ; A \beta 42: 0.75 \pm 0.02, n=8 ; p<0.001$ vs. control, $p<0.0001$ vs. $A \beta 42+T D 139, A \beta 42+$ TD139: $0.85 \pm 0.01, n=10 ; p=0.7034$ vs. control). Inset: Representative example of the autocorrelation function performed on gamma oscillations recorded in the conditions mentioned in A. E) Quantification of gamma oscillation power $(20-80 \mathrm{~Hz})$ showing that the absence of gal3 signaling in 5xFAD mice prevents the gamma power reduction typical of this AD mouse model and results in gamma power similar to WT mice (WT: $1.27 \pm$ $\left.0.18 \times 10^{-09} V^{2}, n=11,5 x F A D: 0.55 \pm 0.03 \times 10^{-09} V^{2}, n=11,5 x F A D-G a l 3 K O: 1.28 \pm 0.25 \times 10^{-09} V^{2}, n=10\right)$. Note that deletion of gal3 in isolation does not affect gamma oscillations power in the Gal3KO mice: $1.34 \pm 0.14 \mathrm{x}$ $10^{-09} \mathrm{~V}^{2}, \mathrm{n}=17$. Statistics performed: Kruskal-Wallis test followed by Dunn's multiple comparisons. A summary is provided in Table S3. F) Summary of peak frequency revealing that 5xFAD mice have slower gamma oscillations (WT: $28.2 \pm 0.61 \mathrm{~Hz}, \mathrm{n}=11,5 x F A D: 24.6 \pm 0.58 \mathrm{~Hz}, \mathrm{n}=11,5 x F A D-G a l 3 K O: 26.4 \pm 1.05 \mathrm{~Hz}, \mathrm{n}=10$, Gal3KO: $26.8 \pm 0.87 \mathrm{~Hz}, \mathrm{n}=17$ ). Statistics performed: ordinary one-way ANOVA followed by Holm-Sidak's multiple comparisons test. A summary is provided in Table S4. Data is presented as a mean \pm SE. Significance levels are shown as * $p<0.05, * * p<0.01, * * * p<0.001$.

\section{Supplementary Files}

This is a list of supplementary files associated with this preprint. Click to download.

- AdditionalFile1.docx

- AdditionalFile2.docx 